\title{
Non-Abelian Topological Order on the Surface of a 3D Topological Superconductor from an Exactly Solved Model
}

\author{
Lukasz Fidkowski, ${ }^{1}$ Xie Chen, ${ }^{2}$ and Ashvin Vishwanath ${ }^{2}$ \\ ${ }^{1}$ Department of Physics and Astronomy, Stony Brook University, Stony Brook, New York 11794-3800, USA \\ ${ }^{2}$ Department of Physics, University of California, Berkeley, California 94720, USA \\ (Received 22 August 2013; revised manuscript received 15 October 2013; published 21 November 2013)
}

\begin{abstract}
Three-dimensional (3D) topological superconductors (TScs) protected by time-reversal $(\mathcal{T})$ symmetry are characterized by gapless Majorana cones on their surface. Free-fermion phases with this symmetry (class DIII) are indexed by an integer $\nu$, of which $\nu=1$ is realized by the $B$ phase of superfluid ${ }^{3} \mathrm{He}$. Previously, it was believed that the surface must be gapless unless time-reversal symmetry is broken. Here, we argue that a fully symmetric and gapped surface is possible in the presence of strong interactions, if a special type of topological order appears on the surface. The topological order realizes $\mathcal{T}$ symmetry in an anomalous way, one that is impossible to achieve in purely two dimensions. For odd $\nu$ TScs, the surface topological order must be non-Abelian. We propose the simplest non-Abelian topological order that contains electronlike excitations, $\mathrm{SO}(3)_{6}$, with four quasiparticles, as a candidate surface state. Remarkably, this theory has a hidden $\mathcal{T}$ invariance that, however, is broken in any two-dimensional realization. By explicitly constructing an exactly soluble Walker-Wang model, we show that it can be realized at the surface of a short-ranged entangled 3D fermionic phase protected by $\mathcal{T}$ symmetry, with bulk electrons transforming as Kramers pairs, i.e. $\mathcal{T}^{2}=-1$ under time reversal. We also propose an Abelian theory, the semion-fermion topological order, to realize an even $\nu$ TSc surface, for which an explicit model is derived using a coupled-layer construction. We argue that this is related to the $\nu=2 \mathrm{TSc}$, and we use this to build candidate surface topological orders for $\nu=4$ and $\nu=8 \mathrm{TScs}$. The latter is equivalent to the three-fermion state, which is the surface topological order of a $\mathbb{Z}_{2}$ bosonic topological phase protected by $\mathcal{T}$ invariance. One particular consequence of this equivalence is that a $\nu=16$ TSc admits a trivially gapped $\mathcal{T}$-symmetric surface.
\end{abstract}

DOI: 10.1103/PhysRevX.3.041016

\section{INTRODUCTION}

Recently, it was pointed out that there exist exotic varieties of insulators and superconductors which form distinct phases of matter. This distinction is based on topological properties and hence falls outside the Ginzburg-Landau-Wilson symmetry-based classification. On the other hand, these distinctions often appear only in the presence of certain symmetries (e.g., time reversal $\mathcal{T}$ ), leading to the terminology "symmetry-protected topological phases" (SPTs). Many such phases can be realized at the level of noninteracting fermions [1-3], and several experimental realizations now exist. Their hallmark signature is the existence of gapless edge or surface modes. The best-known example is the three-dimensional (3D) topological insulator, which is protected by charge conservation and time-reversal symmetry and has a single Dirac cone on its two-dimensional (2D) surface. Another example is the three-dimensional topological superconductor. Here,

Published by the American Physical Society under the terms of the Creative Commons Attribution 3.0 License. Further distribution of this work must maintain attribution to the author(s) and the published article's title, journal citation, and DOI.
Subject Areas: Strongly Correlated Materials, Superfluidity, Topological Insulators

time-reversal symmetry protects gapless Majorana cones at the surface. Within a free-fermion (quadraticHamiltonian) description, different topological phases are labeled by an integer $\nu$. The gaplessness of these surfaces is protected by the symmetry.

While a fairly complete picture exists of free-fermion topological phases [4,5], less is known about topological phases in the presence of interactions. Even if we restrict our attention to SPTs (which may be adiabatically connected to a trivial gapped phase in the absence of symmetry), the qualitatively new phenomena that occur when strong interactions are present are only now beginning to be studied. Two advances in this general area include the result that the free-fermion classification of SPT phases may be reduced in the presence of interactions, as shown for a class of one-dimensional (1D) topological superconductors [6-9] and a class of 2D topological superconductors with $\mathbb{Z}_{2}$ or reflection symmetry [10-13], and the discovery of SPT phases of bosons in different dimensions, that necessarily require interactions for their realization $[5,14-20]$.

An important lesson that emerged from studying interacting bosonic SPT phases is that the surface of a $3 \mathrm{D}$ phase can be gapped without breaking the symmetry (either spontaneously or explicitly) if the surface develops 
topological order. In other words, the system must have deconfined anyons at the gapped, symmetric surface. Moreover, the anyons transform under the symmetries in a way that is disallowed in a strictly two-dimensional system[18]. Explicit examples of such symmetric topologically ordered surfaces have been constructed for 3D bosonic SPT phases [20-22]. The simplest example is a bosonic version of the 3D topological superconductor protected by time-reversal symmetry. In a conventional surface termination, time-reversal symmetry is broken at the surface, leading to a gap. Domain walls between timereversed domains carry chiral edge modes with net chiral edge central charge $c_{-}=8$, and so each domain is associated with half that chiral central charge. However, it was realized that the surface can be gapped while retaining time-reversal symmetry if it acquires the three-fermion [5] $\mathbb{Z}_{2}$-gauge-theory topological order [18]. If this topological order were realized in $2 \mathrm{D}$, it would always break $\mathcal{T}$ symmetry since it is associated with chiral edge states with $c_{-}=4$ (exactly half the value associated with the surface domain walls). However, when realized on the boundary of a 3D system, no edge is present, and the theory can remain time-reversal symmetric. Explicit constructions of such surface phases were given in $[21,22]$.

While 3D SPT phases of bosons still need to be experimentally realized, it is interesting to consider the analogous topologically ordered surface states for the 3D fermionic topological insulators and superconductors, which we know are realized in nature. The topologically ordered surface states provide a rare example of a qualitatively new phenomenon that lies beyond the free-fermion description of these phases. Moreover, as we will see shortly, we will require non-Abelian topological order in some cases. This is a rare example where preserving symmetry requires not just topological order, but also particles with non-Abelian statistics, and may eventually help in realizing these exotic excitations.

In this paper, we propose gapped, topologically ordered, $\mathcal{T}$-invariant terminations for 3D topological superconductors (topological insulators will be discussed in a separate publication). In terms of the free-fermion classification, these correspond to class DIII, where one has a $\mathbb{Z}$ classification $[4,23]$; the $\nu=1$ member of this class is the BalianWerthamer (BW) $[24,25]$ state of the $B$ phase of liquid ${ }^{3} \mathrm{He}$, whose single Majorana cone describing the surface dispersion is protected by time reversal in the noninteracting setting. A principal feature of this surface is that a domain wall between regions of opposite $\mathcal{T}$ breaking is associated with a single chiral Majorana mode, which has chiral central charge $c_{-}=\frac{1}{2}$. Hence, each domain may be associated with $c_{-}=\frac{1}{4}$, something that is impossible in a purely 2D fermionic system without topological order, where $c_{-}$is quantized in units of $\frac{1}{2}$. One strategy is then to look for patterns of fermionic 2D topological order (i.e., those that contain a fundamental fermion that has trivial braiding statistics with every other excitation and can be identified with the electron) and attempt to find a theory with both $c_{-}=\frac{1}{4} \bmod \frac{1}{2}$ and $\mathcal{T}$ symmetry. Fortunately, this approach turns out to be fruitful because 2D fermionic topological orders are extremely constrained.

First, we can rule out Abelian theories, for the following reason: The fusion-braiding universality class of any fermionic Abelian topological order can be realized in a $K$-matrix formulation, which has integral chiral central charge. Adding in layers of a $p+i p$ superconductorwhich does not change the anyon spectrum-can only change this chiral central charge in integer multiples of $\frac{1}{2}$ and can never generate $c_{-}=\frac{1}{4} \bmod \frac{1}{2}$. A well-known nonAbelian example is the Moore-Read Pfaffian state [26], but it is not $\mathcal{T}$ invariant nor does it have the correct $c_{-}$[27]. However, the Pfaffian, with 12 quasiparticle types, is not the simplest non-Abelian fermionic theory. Indeed, from a classification standpoint, the smallest such theory contains only four quasiparticle types: In addition to the trivial particle and the electron, there is a self-semion $s$ and its time-reversed partner $\tilde{s}$. This is the integral spin subtheory of $\mathrm{SU}(2)_{6}$, equivalent to $\mathrm{SO}(3)_{6}$ [28,29]. Serendipitously, $\mathrm{SU}(2)_{6}$ has $c_{-}=\frac{9}{4}=\frac{1}{4} \bmod \frac{1}{2}$, and the braiding and fusion rules of its integral-spin subtheory are invariant under a $\mathcal{T}$ symmetry that exchanges $s$ and $\tilde{s}$. A strict $2 \mathrm{D}$ realization of this phase should break $\mathcal{T}$ as we argue below, based on the nontrivial-edge chiral central charge $c_{-}=\frac{9}{4}$ in one realization. However, it is conceivable that it may appear as a $\mathcal{T}$-invariant surface state of a 3D bulk, based on the statistics of the excitations. In that case, one cannot interrogate the edge content, and hence a hidden $\mathcal{T}$ symmetry may exist. We substantiate this claim by explicitly constructing a $3 \mathrm{D} \mathcal{T}$-symmetric model whose surface displays the $\mathrm{SO}(3)_{6}$ topological order.

Our central tool is the Walker-Wang (WW) construction [30,31], which, in essence, converts a given surface topological order into a prescription for the bulk wave function (similar to the connection, one dimension lower, between quantum Hall wave functions and edge conformal field theories). The construction also provides an exactly soluble model to realize this wave function and surface topological order-so we know explicitly that it can be realized at the surface of an appropriate 3D system. Moreover, in the case of fermionic topological phases, it allows us to fix the transformation law for fermions under the protecting symmetry. Here, we will see that the electrons must transform projectively, i.e., with $\mathcal{T}^{2}=-1$ under time-reversal symmetry. For constructing the exactly soluble 3D models, one is given the surface topological order, specified in terms of quasiparticle labels and fusion and braiding rules, collectively denoted $\mathcal{B}$ (mathematically, a premodular unitary category). When the only particle that braids trivially with everything in $\mathcal{B}$ is the identity-the so-called modular case-the WW models realize a confined $3+$ 1-dimensional phase, with topological order $\mathcal{B}$ on the 
surface. Although the bulk is trivial, these models sometimes naturally allow for an incorporation of symmetry that can protect the surface topological order and hence result in a nontrivial 3D bosonic SPT [22]. This strategy was successfully applied to construct WW models that realize the three-fermion surface topological order with time-reversal symmetry, which realizes a 3D bosonic SPT phase [22]. In the present case, however, the input $\mathcal{B}=$ $\mathrm{SO}(3)_{6}$ contains the electron, which has trivial braiding statistics with all other excitations. We will see that this leads to a 3D WW model whose only bulk deconfined excitation is a $\mathcal{T}^{2}=-1$ fermion-the electron-and whose surface realizes $\mathrm{SO}(3)_{6}$. We note a minor caveat here. Since it is convenient to work entirely in terms of a bosonic WW model, rather than introducing fundamental fermions (electrons), the deconfined bulk fermions carry $\mathbb{Z}_{2}$ gauge charge and realize the $\mathbb{Z}_{2}$-gauged $3 \mathrm{D}$ topological superconductor. Ungauging this theory by suppressing $\mathbb{Z}_{2}$ flux loops is straightforward and yields the topological superconductor.

Finally, we discuss Abelian topological orders that are candidates for surfaces of even $\nu$ topological superconductors. Specifically, we propose three such topological orders - the semion-fermion model, the doubled semionfermion model, and the fermionization of the bosonic SPT. The semion-fermion model is simply $U(1)_{2}$ times $\{1, f\}$, where $f$ is the electron; however, time reversal acts in a nontrivial way, exchanging the semion $s$ with $s f$. We argue that this theory cannot be realized in $2 \mathrm{D}$ with $\mathcal{T}$ symmetry, but it can appear on the surface of a 3D topological phase. In addition to a Walker-Wang model, we provide a coupled-layer construction of this phase. An important point is that there are two varieties of the semion-fermion model, distinguished by the action of $\mathcal{T}^{2}$. These models are opposite to each other in the sense that placing one on top of the other allows one to condense everything and yields a trivial $\mathcal{T}$-symmetric theory; i.e., one corresponds to $\nu$ and the other to $-\nu$. However, two copies of the same variety yield the double semion-fermion model. Two copies of the latter yield the three-fermion $\mathbb{Z}_{2}$ gauge theory discussed above in the context of the bosonic SPT, times $\{1, f\}$. We argue that this fermionic theory cannot be realized in $2 \mathrm{D}$ with $\mathcal{T}$ symmetry either, but two copies of it can be. It has been conjectured previously [32] that the noninteracting $\mathbb{Z}$ classification of 3D topological superconductors breaks down to $\mathbb{Z}_{16}$; if the $\mathrm{SO}(3)_{6}$ and semion-fermion topological superconductors we construct adiabatically connect to free-fermion topological superconductors, then the three Abelian topological orders must correspond to $\nu=2 \mathrm{mod}$ $4, \nu=4 \bmod 8$, and $\nu=8 \bmod 16$. Taken together, these would then give topological terminations for all free-fermion topological superconductors.

This paper is organized as follows. In Sec. II, we treat the $\mathrm{SO}(3)_{6}$ topological order, corresponding to $\nu=1 \bmod 2$. An intuitive discussion of why it can be realized at the surface of a 3D $\mathcal{T}$ SPT of electrons is presented in Sec. II B, and the Walker-Wang construction is discussed in the following two subsections. Next, in Sec. III, the Abelian topological orders are discussed, starting with the semion-fermion state, for which we also provide a coupled-layer construction. We provide an argument for the semion-fermion state being realized at the surface of a $\nu=2 \bmod 4 \mathrm{TSc}$, and then derive the Abelian topological orders corresponding to $\nu=$ $4 \bmod 8$ and $\nu=8 \bmod 16$. The Conclusion connects our results with the classification of fermionic TScs and comments on future directions; the appendixes contain a construction of a $2 \mathrm{D} \mathbb{Z}_{2}$ gauge theory in which $\mathcal{T}$ exchanges the $\mathbb{Z}_{2}$ charge $e$ and the $\mathbb{Z}_{2}$ flux $m$ particles, and a discussion of the stability of bosonic SPT phases protected by $\mathcal{T}$, in the presence of fundamental electrons.

\section{SO $(3)_{6}$ TOPOLOGICAL ORDER AND WALKER-WANG CONSTRUCTION OF THE 3D PHASE}

\section{A. $\operatorname{SO}(3)_{6}$ topological order of surface state}

Before delving into the construction of the WalkerWang model, let us describe the fusion and braiding properties of $\mathrm{SO}(3)_{6}$. A useful viewpoint on this phase is to begin with the well-known topological order $\mathrm{SU}(2)_{6}$ [33], which is a bosonic Read-Rezayi state [29,34] with six quasiparticles labeled by spins $j \in\left\{0, \frac{1}{2}, 1, \frac{3}{2}, 2, \frac{5}{2}, 3\right\}$, and it is described by the $\mathrm{SU}(2)_{k=6}$ Wess-Zumino-Witten chiral edge theory with chiral central charge $c_{-}=\frac{9}{4}$. The topological spins of the quasiparticles are $\theta_{j}=e^{i 2 \pi \frac{j(j+1)}{8}}$. Thus, $j=1$ and $j=2$ particles are self-semions, with topological spins $\pm i$, while the $j=3$ particle is a fermion. However, the fermion has mutual statistics with the half-integer spin particles. The latter can be eliminated if we introduce fundamental fermions (electrons) into the theory and condense the bound state of the $j=3$ particle and the electron. This bound state can be condensed since it is a self-boson. However, since it has mutual statistics with the half-integer spin particles $j=\frac{1}{2}, \frac{3}{2}, \frac{5}{2}$, they are confined, and one is left with just the integer spin particles. Of these, the fourth particle $j=3$ has trivial braiding statistics with the remaining excitations, and it is identified as the electron (note that this results in a nonmodular theory, as in any topological order that contains fundamental fermions). Since only the integer representations of the spins remain in the final theory, we call it $\mathrm{SO}(3)_{6}$ topological order [35]. The condensation process that converts the $\mathrm{SU}(2)_{6}$ to $\mathrm{SO}(3)_{6}$ does not change the edge central charge, and hence, the final theory is also expected to have $c_{-}=\frac{9}{4}$. We henceforth denote the two self-semions by $s, \tilde{s}$, and the fermion by $e:\{0,1,2,3\} \rightarrow$ $\{1, s, \tilde{s}, e\}$. Their fusion rules and topological spins are shown in Fig. 1.

In our analysis, we will need more information about the fusion and braiding, however: The data that enter the 


\begin{tabular}{|cc|}
\hline Fusion rules : & Quantum dimensions and topological spins : \\
$s \times s=\tilde{s} \times \tilde{s}=1+s+\tilde{s}$ & $d_{1}=d_{e}=1, d_{s}=d_{\tilde{s}}=1+\sqrt{2}$ \\
$s \times \tilde{s}=e+s+\tilde{s}$ & $\theta_{1}=1, \theta_{e}=-1, \theta_{s}=i, \theta_{\tilde{s}}=-i$ \\
$s \times e=\tilde{s} \quad \tilde{s} \times e=s$ & $F$ symbols : \\
$e \times e=1$ & {$\left[F_{s}^{e, \tilde{s}, s}\right]_{s, \tilde{s}}=\left[F_{s}^{e, s, \tilde{s}}\right]_{\tilde{s}, \tilde{s}}=-1$} \\
$R$ symbols : & {$\left[F_{s}^{\tilde{s}, \tilde{s}, s}\right]_{1, s}=\left[F_{s}^{s, s, s}\right]_{1, \tilde{s}}=\left[F_{\tilde{s}}^{s, s, \tilde{s}}\right]_{1, s}=$} \\
$R_{0}^{s, s}=e^{-\pi i / 2}$ & {$\left[F_{s}^{s, \tilde{s}, \tilde{s}}\right]_{e, s}=\left[F_{s}^{\tilde{s}, s, \tilde{s}}\right]_{e, s}=\left[F_{\tilde{s}}^{\tilde{s}, \tilde{s}}\right]_{1, \tilde{s}}=(1+\sqrt{2})^{-\frac{1}{2}}$} \\
$R_{s}^{s, s}=R_{\tilde{s}}^{s, \tilde{s}}=e^{3 \pi i / 4}$ & {$\left[F_{s}^{s, s, s}\right]_{s, s}=\left[F_{s}^{\tilde{s}, s, \tilde{s}}\right]_{s, s}=\left[F_{s}^{\tilde{s}, \tilde{s}, s}\right]_{\tilde{s}, s}=(2+\sqrt{2})^{-1}$} \\
$R_{\tilde{s}}^{s, s}=e^{\pi i / 4}$ & {$\left[F_{s}^{\tilde{s}, s, s}\right]_{s, s}=\left[F_{s}^{\tilde{s}, \tilde{s}, s}\right]_{s, s}=-1 / \sqrt{2}$} \\
$R_{s}^{s, \tilde{s}}=R_{\tilde{s}}^{\tilde{s}, \tilde{s}}=e^{-3 \pi i / 4}$ & $F_{\mathrm{symbols} \mathrm{that} \operatorname{change~sign}}$ \\
$R_{e}^{s, \tilde{s}}=R_{1}^{\tilde{s}, \tilde{s}}=e^{\pi i / 2}$ & under s $\leftrightarrow \tilde{\mathrm{s}}:$ \\
$R_{s}^{\tilde{s}, \tilde{s}}=e^{-\pi i / 4}$ & {$\left[F_{\tilde{s}}^{e, s, \tilde{s}}\right]_{\tilde{s}, s},\left[F_{\tilde{s}}^{e, \tilde{s}, s}\right]_{s, s},\left[F_{s}^{s, s, s}\right]_{1, s},\left[F_{s}^{\tilde{s}, \tilde{s}, s}\right]_{1, s}$,} \\
$R_{\tilde{s}}^{s, e}=R_{1}^{e, e}=-1$ & {$\left[F_{s}^{s, s, s}\right]_{1, \tilde{s}},\left[F_{\tilde{s}}^{s, s, \tilde{s}}\right]_{1, s},\left[F_{s}^{\tilde{s}, \tilde{s}, s}\right]_{s, s}\left[F_{s}^{\tilde{s}, \tilde{s}, s}\right]_{s, s}$} \\
and & and all partners under s $\leftrightarrow \tilde{\mathrm{s}}$. \\
$R_{a}^{b, c}=R_{a}^{c, b}$ &
\end{tabular}

FIG. 1. Braiding statistics and fusion data for $\mathrm{SO}(3)_{6}$.

WW Hamiltonian require the $F$ and $R$ symbols, which describe the associativity of fusion (a quantum analogue of Clebsch-Gordon coefficients) and exchange of an arbitrary pair of quasiparticles, respectively, and uniquely determine $\mathrm{SO}(3)_{6}$ as a premodular category [29]. For the definition of the $F$ symbols, see Fig. 2, which also contains some of their symmetries. All of the nontrivial $F$ symbols (i.e., those not equal to 1) can be obtained from those in Fig. 1 by using compositions of these symmetries, together with their invariance up to a sign under $s \leftrightarrow \tilde{s}$. A representative set of $F$ symbols that change sign under this exchange are also shown in Fig. 1.

$$
\begin{aligned}
& \overbrace{j_{1} j_{2} j_{3}}^{j}=\left[F_{j}^{j_{1}, j_{2}, j_{3}}\right]_{j_{12}, j_{23}} \\
& {\left[F_{j}^{j_{1}, j_{2}, j_{3}}\right]_{j_{12}, j_{23}}^{j_{23}}=\left[F_{j_{3}}^{j_{2}, j_{1}, j}\right]_{j_{12}, j_{23}}^{j}=} \\
& {\left[F_{j_{2}}^{j_{1}, j_{3}}\right]_{j_{23}, j_{12}}=\sqrt{\frac{d_{j_{12} d_{j_{23}}}^{d_{j_{2}} d_{j}}}{j_{12}}\left[F_{j_{23}}^{j_{1}, j_{12}, j_{3}}\right]_{j_{2}, j}}}
\end{aligned}
$$

FIG. 2. Graphical definition of the $F$ symbol, together with some identities satisfied by $F$.

\section{B. Time-reversal symmetry and $\operatorname{SO}(3)_{6}$ topological order}

We first elaborate on the question of time-reversal symmetry and the $\mathrm{SO}(3)_{6}$ topological order. As discussed in the overview, since this topological order is obtained by condensing particles in the $\mathrm{SU}(2)_{6}$ theory, which is modular and has an edge central charge $c_{-}=\frac{9}{4}$, we expect the same edge central charge in at least some realizations of this phase. Could one potentially realize the same $\mathrm{SO}(3)_{6}$ topological order with a trivial edge? We now argue that it is impossible to realize this topological order in $2 \mathrm{D}$ without a chiral edge mode. This fact guarantees that it cannot be realized in a $2 \mathrm{D}$ system with $\mathcal{T}$ symmetry, and thus, if realized on the surface of a $3 \mathrm{D} \mathcal{T}$ symmetric system, it defines a 3D topological phase. For modular topological orders, where all nontrivial quasiparticles have nontrivial braiding statistics with at least one other particle, a powerful formula relates the topological properties to the chiral edge central charge $c_{-} \bmod 8$ [5]:

$$
\frac{1}{\mathcal{D}} \sum_{a} d_{a}^{2} \theta_{a}=e^{i 2 \pi c_{-} / 8}
$$

where $d_{a}(\mathcal{D})$ are the individual (total) quantum dimensions of the quasiparticles. Unfortunately, we cannot directly apply this formula since we have a nonmodular theory (the electron has trivial braiding with all quasiparticles). In fact, we can easily see that the edge central charge can be changed by units of $c_{-}=\frac{1}{2}$ without affecting the topological order, simply by putting the electrons in a 
$p \pm i p$ topological superconductor phase, which suggests that the edge of $\mathrm{SO}(3)_{6}$ is necessarily chiral. This result can be further argued as follows. Imagine that there is also a realization of the same topological order with a $c_{-}=0$ edge, and consider it in conjunction with the existing $c_{-}=\frac{9}{4}$ realization. Then, we could perform a reflection on the first phase and combine it with the second. In this way, we will have realized a quantum double model, which can always be confined by condensing a bosonic quasiparticle. In this process, the edge central charge will not change and will remain $c_{-}=\frac{9}{4}$. However, we now have eliminated all topological order. A fermionic system with no topological order must have half-integer quantized edge central charge, so we have arrived at a contradiction. Therefore, there cannot be a 2D system with $\mathrm{SO}(3)_{6}$ topological order and without a chiral edge state.

Let us now consider time reversal purely at the level of fusion and braiding rules. An examination of the topological spins in our theory (Fig. 1) suggests that it is timereversal invariant under the exchange $s \leftrightarrow \tilde{s}$. Indeed, such an exchange, together with complex conjugation, gives a set of $F$ and $R$ symbols that must be gauge equivalent to the original ones because $\mathrm{SO}(3)_{6}$ is the unique theory with these topological spins and fusion rules. However, $\mathcal{T}$ invariance of the WW Hamiltonian (constructed in the next section) requires more: We will need a time-reversal transformation law that leaves the $F$ and $R$ symbols exactly invariant, not just invariant up to gauge transformation. Quick examination of $\left[F_{e}^{s, s, s}\right]_{\tilde{s}, \tilde{s}}$ and $\left[F_{e}^{\tilde{s}, \tilde{s}, \tilde{s}}\right]_{s, s}$ shows that there is no gauge in which such a transformation law takes the simple form $s \leftrightarrow \tilde{s}$ followed by complex conjugationthis is because these two $F$ symbols differ by a sign in our gauge and transform by complex-conjugate phases under a gauge transform, so they cannot be complex conjugates in any gauge. However, the following, more general $\mathcal{T}$ transformation law does work and, in fact, forces $\mathcal{T}^{2}=-1$ on the electrons: We define $\mathcal{T}$ as the operation that exchanges $s \leftrightarrow \tilde{s}$, complex conjugates, and multiplies by certain phase factors $\alpha_{c}^{a, b}$ (see below) associated with the fusion spaces $V_{c}^{a, b}$ in our anyon theory [36]. It is this $\mathcal{T}$ transformation that commutes with all of the $F$ and $R$ symbols; link invariants (i.e., braiding amplitudes) computed in the corresponding picture calculus are then invariant under it.

The phase factors $\alpha_{c}^{a, b}$ are defined as follows. For vertices of type $(s, \tilde{s}, e), \alpha_{c}^{a, b}$ is $\pm i$ depending on the sign of the permutation that takes $(s, \tilde{s}, e)$ to $(a, b, c)$. Specifically,

$$
\alpha_{e}^{s, \tilde{s}}=\alpha_{s}^{\tilde{s}, e}=\alpha_{\tilde{s}}^{e, s}=i, \quad \alpha_{\tilde{s}}^{s, e}=\alpha_{s}^{e, \tilde{s}}=\alpha_{e}^{\tilde{s}, s}=-i .
$$

For vertices at which one or more of $a, b, c$ is the trivial anyon 1 , we set $\alpha_{c}^{a, b}=1$. In the remaining vertices $a, b, c$ are all either $s$ or $\tilde{s}$. We take

$$
\alpha_{s}^{s, s}=\alpha_{\tilde{s}}^{\tilde{s} \tilde{s}}=i
$$

and $\alpha_{c}^{a, b}=-i$ when $(a, b, c)$ consists of some permutation of two $s$ 's and one $\tilde{s}$ or vice versa.

With this definition, $\mathcal{T}$ commutes with $F$. Since in our gauge the $F$ symbols are all real, this commutation reads

$$
\alpha_{n}^{j, k} \alpha_{l}^{i, n}\left[F_{l}^{i, j, k}\right]_{m, n}=\left[F_{\tilde{l}}^{\tilde{i} \tilde{j}, \tilde{k}}\right]_{\tilde{m}, \tilde{n}} \alpha_{m}^{i, j} \alpha_{l}^{m, k},
$$

where $i \rightarrow \tilde{i}$ is just the permutation that fixes $i=1$, $e$ and exchanges $i=s, \tilde{s}$. $\mathcal{T}$ also commutes with $R$ :

$$
\alpha_{k}^{j, i}\left(R_{k}^{i, j}\right)^{*}=R_{\tilde{k}}^{\tilde{i}, \tilde{j}} \alpha_{k}^{i, j} .
$$

These two relations are sufficient to show that Wilson lines computed in the corresponding picture calculus are invariant under $\mathcal{T}$. Note that $\left(\alpha_{\tilde{k}}^{\tilde{i}, \tilde{j}}\right)\left(\alpha_{k}^{i, j}\right)^{*}=1$ for all vertex types, except those where $(i, j, k)$ is a permutation of $(s, \tilde{s}, e)$, for which $\left(\alpha_{\tilde{k}}^{\tilde{i}, \tilde{j}}\right)\left(\alpha_{k}^{i, j}\right)^{*}=-1$. This means that $\mathcal{T}^{2}$ fixes all particle types and gives minus signs to $(s, \tilde{s}, e)$ vertices. In any string net that respects the fusion rules, there are an even number of such vertices, so $\mathcal{T}^{2}$ acts as the identity on them. In the exactly solved Walker-Wang model of the next section, this fact translates to the many-body ground state being invariant under $\mathcal{T}$, with $\mathcal{T}^{2}=1$. However, string-net configurations of the Walker-Wang model also include fusion-rule violating vertices such as $(1,1, e)$; a consistent definition of time reversal that includes these will require them to have $\mathcal{T}^{2}=-1$, which will necessitate decorating the Walker-Wang model with additional spin $1 / 2$ 's (Kramers doublets). In the next section, we will see how to do this by binding Haldane chains to the $e$ links of the model. Ultimately, this ensures that the fundamental fermions in the theory (the electrons) must transform under time reversal as $\mathcal{T}^{2}=-1$. Since this is an important point, we elaborate on its origin in a more intuitive way below.

Emergence of $\mathcal{T}^{2}=-1$ for fermions in the WalkerWang model.-Let us briefly review the idea behind the Walker-Wang construction in order to make this point. The Hilbert space, defined on the links of the 3D lattice model, contains a state for each quasiparticle in the theory-in this case, there are four states per link, labeled by the four particle types. The ground-state wave function is a quantum superposition of loops labeled by these four indices (or four colors). At the vertices (we consider a trivalent lattice for simplicity - it is always possible to deform a lattice such as a cubic lattice into a trivalent one by splitting vertices), the loops are allowed to branch and combine according to the fusion rules. The amplitudes for these loop configurations are determined by imagining them as space-time Wilson loops of the $2+1 \mathrm{D}$ TQFT representing the surface topological order and calculating the quantum amplitudes within that theory. Thus, $\Psi_{3 \mathrm{D}}(C)=$ $\langle W(C)\rangle_{2+1 \mathrm{TQFT}}$. In practice, these amplitudes are implemented by writing down a parent Hamiltonian. Consider a part of the ground-state wave function as shown in the topleft diagram of Fig. 3. The $s$ and $\tilde{s}$ particles of the theory, which have semion and "antisemion" self-statistics, fuse 

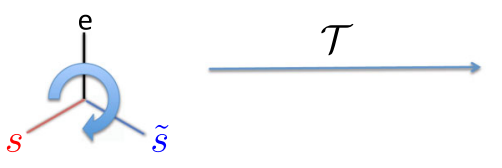

$\Psi$

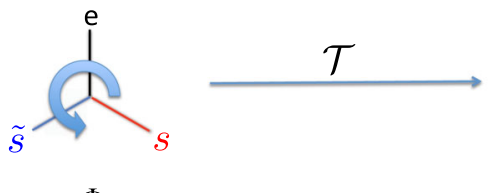

$\Phi$

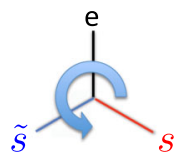

i $\Psi^{*}$

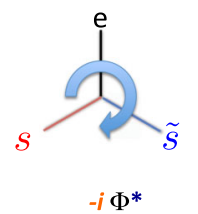

$\Phi^{*}$

$$
\mathcal{T}^{2}=-1
$$

FIG. 3. Origin of $\mathcal{T}^{2}=-1$ in Walker-Wang models of $\mathrm{SO}(3)_{6}$ surface topological order. The additional phase factor shown in red depends on the orientation of the vertices and is required to ensure time-reversal symmetry. This leads to $\mathcal{T}^{2}=-1$ for fermions in this model.

to give the electron $e$. Say this has an associated amplitude $\Psi$. Then, in order to preserve time-reversal symmetry, one needs to not only complex conjugate this amplitude, but also multiply by a phase factor $\pm i$. Only then does the transformation commute with the $\mathcal{R}$ moves and produce a time-reversal symmetric wave function. The choice of phase factor is fixed by the orientation of the vertexe.g., if we choose $i$ for the vertex, where the $\{e, \tilde{s}, s\}$ appear moving anticlockwise continuously, we must choose $-i$ for the vertex of the opposite sense, shown on the bottom-left diagram. Since time reversal exchanges $s$ and $\tilde{s}$, and hence these two vertices, we have the sequence $\Psi \rightarrow^{\mathcal{T}} i \Psi^{*} \rightarrow^{\mathcal{T}}-i\left(i \Psi^{*}\right)^{*}=-\Psi$ and thus $\mathcal{T}^{2}=-1$. Since this vertex results in the creation of a fermion, we will ultimately associate this transformation law with the fermions in the theory. Note that in the Walker-Wang models, clockwise and counterclockwise orientations are fixed since the projection of the 3D lattice on the 2D plane is fixed $a$ priori in order to define the model. Hence, one can imagine that a crystal axis is picked when defining the models, and the remaining sense along the axis, required to define a spinning particle, is provided by the permutation of the three labels.

The time-reversal symmetry described above assigns a phase factor that depends jointly on the state of three bonds that meet at a vertex. Such a non-onsite transformation, i.e., one that does not act independently on the physical variables that reside at links, is not a fully satisfactory implementation of symmetry. This situation is readily fixed by attaching additional link variables that are put into a "Haldane chain" phase protected by time-reversal symmetry, along the $e$ strings. When these strings end (for example, by splitting into an $s, \tilde{s}$ pair, as in Fig. 3), the spin- $1 / 2$ excitation generated at the ends automatically provides the requisite phase factors to keep the state invariant under time reversal. Furthermore, the vertex-term-violating configurations mentioned previously are also made timereversal symmetric by this construction.

Variants of $\mathcal{T}$ action on $\mathrm{SO}(3)_{6}$.- Another important point is that if $\mathrm{SO}(3)_{6}$ indeed corresponds to some odd- $\nu$ topological superconductor, then one would expect its parity conjugate-i.e., a flipped version of $\mathrm{SO}(3)_{6}$ - to be distinct and correspond to $-\nu$. We address this issue for the (easier) Abelian semion-fermion theory in the following section, but leave the case of $\mathrm{SO}(3)_{6}$ to future work.

\section{Review of standard Walker-Wang construction}

We now construct our exactly soluble model. First, we review the standard Walker-Wang construction [30,31], for the specific case of the premodular category $\mathcal{B}=\mathrm{SO}(3)_{6}$. This model will have $\mathrm{SO}(3)_{6}$ topological order on the surface, and its only deconfined bulk excitation will be a fermion. Then, we describe how to extend it by "gluing Haldane chains" [39] to the $e$ lines; the resulting model will be $\mathcal{T}$ invariant under a natural onsite $\mathcal{T}$ symmetry, with the bulk deconfined fermion-the electron-carrying $\mathcal{T}^{2}=-1$.

Informally, the states in the WW model are string nets obeying the fusion rules of $\mathcal{B}$. The Hamiltonian is engineered in such a way that the ground state consists of a superposition of such string nets, with amplitudes equal to their evaluation in the picture calculus of $\mathcal{B}$. It is important to note that the WW models work with a particular planar projection of the 3D lattice, yielding a natural choice of framing. Only the deconfined strings correspond to the socalled symmetric center $Z(\mathcal{B})$ : quasiparticles in $\mathcal{B}$ that have trivial braiding with all other quasiparticles. In our case, these are just the electrons. Furthermore, the statistics of the bulk deconfined excitations are just those of $Z(\mathcal{B})$, so our bulk deconfined quasiparticle is indeed a fermion $[30,40]$.

In order to explicitly describe the Walker-Wang model, we closely follow Ref. [30] and refer the reader there for further details. We start with a planar projection of a trivalent resolution of the cubic lattice, as in Fig. 4. The links are labeled with the quasiparticle types of $\mathrm{SO}(3)_{6}$.

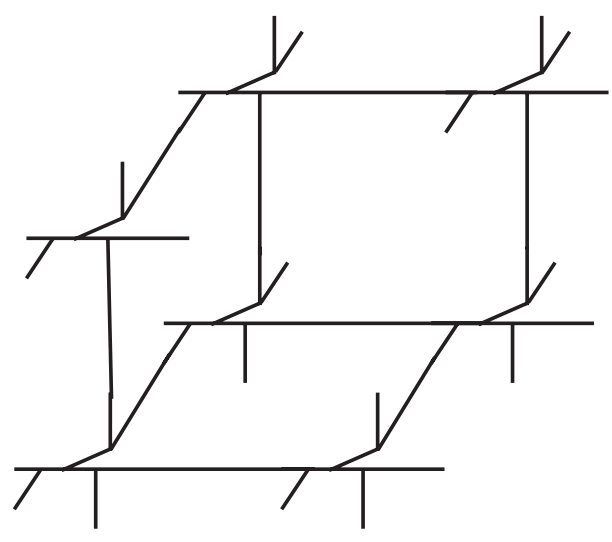

FIG. 4. Trivalent resolution of the cubic lattice [30]. 


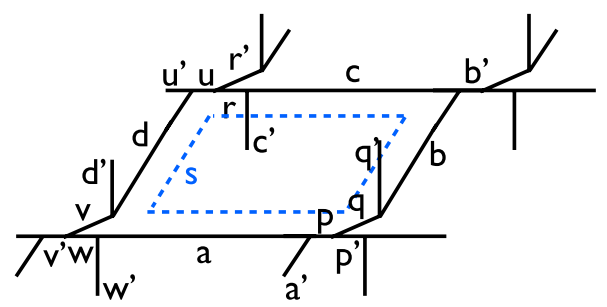

FIG. 5. Plaquette term in the Walker-Wang model (taken from Ref. [30]).

The Hamiltonian enforces fusion rules at the vertices and contains some plaquette terms. For any quasiparticle $s$ and plaquette $P$ (Fig. 5), there is a term in the Hamiltonian which acts on the links of that plaquette, labeled in Fig. 5. This term can change the labels of these links and can also depend on the labels of adjoining links, which have primes on them (but cannot change these). Explicitly, the matrix element between a state with plaquette links $(a b c d p q r u v w)$ and $\left(a^{\prime \prime} b^{\prime \prime} c^{\prime \prime} d^{\prime \prime} p^{\prime \prime} q^{\prime \prime} r^{\prime \prime} u^{\prime \prime} v^{\prime \prime} w^{\prime \prime}\right)$ is

$$
\begin{aligned}
\left(B_{P}^{s}\right)_{a^{\prime \prime}, \ldots, w^{\prime \prime}}^{a_{1}, \ldots, w}= & R_{q}^{q^{\prime} b}\left(R_{c}^{c^{\prime} r}\right)^{*}\left(R_{q^{\prime \prime}}^{q^{\prime} b^{\prime \prime}}\right)^{*} R_{c^{\prime \prime}}^{c^{\prime} r^{\prime \prime}}\left[F_{a^{\prime}}^{a^{\prime \prime}, s, p}\right]_{a, p^{\prime \prime}} \\
& \times\left[F_{p^{\prime}}^{p^{\prime \prime}, s, q}\right]_{p, q^{\prime \prime}}\left[F_{q^{q^{\prime}}}^{q^{\prime \prime}, s, b}\right]_{q, b^{\prime \prime}}\left[F_{b^{\prime}}^{b^{\prime \prime}, s, c}\right]_{b, c^{\prime \prime}} \\
& \times\left[F_{c^{\prime}}^{c^{\prime \prime}, s, r}\right]_{c, r^{\prime \prime}}\left[F_{r^{\prime}}^{r^{\prime \prime}, s, u}\right]_{r, u^{\prime \prime}}\left[F_{u^{\prime}}^{u^{\prime \prime}, s, d}\right]_{u, d^{\prime \prime}} \\
& \times\left[F_{d^{\prime}}^{d^{\prime \prime}, s, v}\right]_{d, v^{\prime \prime}}\left[F_{v^{\prime}}^{v^{\prime \prime}, s, w}\right]_{v, w^{\prime \prime}}\left[F_{w^{\prime}}^{w^{\prime \prime}, s, a}\right]_{w, a^{\prime \prime}}
\end{aligned}
$$

The intuition behind this complicated-looking term is that it fuses in the loop $s$ to the skeleton of the plaquette using multiple $F$ moves, but in the process of doing so, it must use $R$ symbols to temporarily displace certain links $\left(c^{\prime}\right.$ and $q^{\prime}$ in Fig. 5). The Hamiltonian then contains a sum of all these plaquette terms, weighted by the quantum dimensions $d_{s}$. It is possible to check that all of these terms commute, and the result is a model that satisfies the properties described above-again, we refer the interested reader to $[30,31]$ for more details. We have thus constructed an exactly solved model that explicitly realizes the $\mathrm{SO}(3)_{6}$ theory on its surface.

\section{Improved Walker-Wang model-onsite $\mathcal{T}$ symmetry and ungauging the bulk topological order}

\section{Exactly soluble model with onsite $\mathcal{T}$ symmetry}

One way to make the model of Sec. II C time-reversal invariant is by defining the $\mathcal{T}$ operator to act by $s \leftrightarrow \tilde{s}$ on link labels and the phase factors $\alpha_{k}^{i, j}$ on vertices. As discussed in Sec. II B, this commutes with the $F$ and $R$ symbols and, hence, with the Walker-Wang Hamiltonian. However, in order to interpret this as the time-reversal invariance of the $\mathbb{Z}_{2}$-gauged topological superconductor, we have to vary this definition slightly.

To see why, note that in the Walker-Wang model, one has to make some choice about how it will act on vertices that violate the fusion rules. For example, consider a configuration of an electron string that terminates at a $(1,1, e)$ fusion-rule violating vertex on one end, and at an $(s, \tilde{s}, e)$ fusion-rule respecting vertex on the other. This is certainly not a low-energy state, with respect to the vertex or plaquette terms, but it is an allowed Hilbert-space configuration. Unless we change the $(1,1, e)$ vertex into a Kramers pair, which requires introducing extra spin- $1 / 2$ degrees of freedom not present in the original WalkerWang model, we will have $\mathcal{T}^{2}=-1$ on this configuration [from the one $(s, \tilde{s}, e)$ vertex]. But this cannot happen in a finite $\mathbb{Z}_{2}$-gauged topological superconductor, where the odd-charge sector has been projected out. Hence, we are forced to introduce some additional spin- $1 / 2$ degrees of freedom in order to have a $\mathcal{T}$ symmetry consistent with that of a $\mathbb{Z}_{2}$-gauged topological superconductor-this is another argument for the introduction of additional spin- $1 / 2$ degrees of freedom.

Specifically, we proceed as follows: Decorate each link with four extra states, to be thought of as two spin 1/2's, one associated with each of the two vertices adjacent to the link. Then, add a term $H_{V}$ to the Hamiltonian that projects these into a singlet, unless the link is labeled with an $e$, in which case it forces the two spin 1/2's into singlets with spin 1/2's from other $e$ lines adjacent to those vertices. If there are none (or if a total of $3 e$ lines meet at the vertex), there will be an unpaired spin $1 / 2$ there. This result is very similar to the construction in Ref. [39], and it has the effect of binding Haldane chains to the $e$ lines. At a $(s, \tilde{s}, e)$ vertex, we also add a term to $H_{V}$ that energetically prefers either the up spin or the down spin, depending on the sign of the permutation that takes $(s, \tilde{s}, e)$ into a counterclockwise labeling of the three links adjoining the vertex (see Fig. 6). Finally, we modify the plaquette terms in the original WW Hamiltonian in such a way that they move between low-energy spin configurations-for details, see Appendix C.

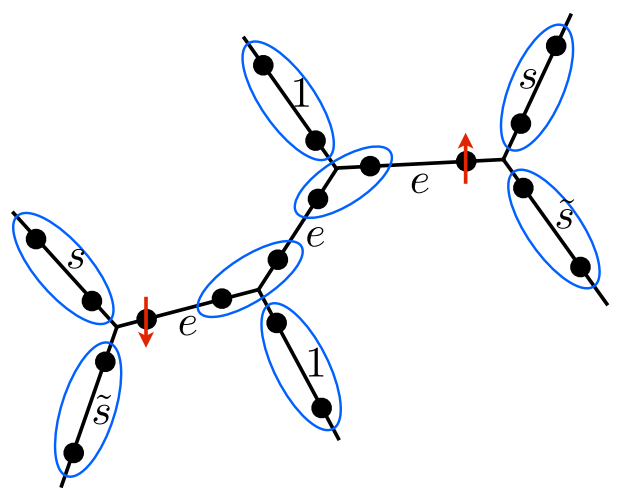

FIG. 6. Sample spin configuration in the decorated WalkerWang model. Black dots represent spin 1/2's, and blue ellipses represent spin singlets. The leftmost vertex has a counterclockwise $(e, s, \tilde{s})$ ordering of labels and so prefers a down spin, whereas at the rightmost vertex, the counterclockwise label ordering is $(e, \tilde{s}, s)$ and an up spin is preferred (red arrow). 
The Hamiltonian constructed in Appendix C commutes with a global symmetry $\mathcal{T}$ that acts on the spin $1 / 2$ 's in the usual way, by complex conjugation followed by multiplication by $\sigma^{y}$ (note the extra factor of $i$ in our definition-it can be eliminated by a gauge transform if desired; it is only $\mathcal{T}^{2}=-1$ that is important), and on the Walker-Wang labels by exchanging $s \leftrightarrow \tilde{s}$ and applying the phase factors $\alpha_{c}^{a, b}$ on vertices at which all of the $a, b, c$ are either $s$ or $\tilde{s}$. Although this is not a true onsite action, it can be made so by a suitable gauge transformation [42]. In this system, all endpoints of $e$ lines effectively contribute a minus sign to $\mathcal{T}^{2}$, and since the number of such endpoints is even in any configuration, the Hilbert space has $\mathcal{T}^{2}=1$.

\section{Ungauging the bulk $\mathbb{Z}_{2}$ topological order}

A second point has to do with the nature of the bulk phase in the WW model, when the surface topological order contains an electron, i.e., a fermion with trivial braiding statistics with all other excitations. In this case, the fermionic excitation is deconfined in the bulk. However, note that all the degrees of freedom entering the microscopic WW model are bosonic, since they simply consist of local "qbits" defined on the links of the lattice. Hence, in order to produce fermionic excitations in the bulk, one must have bulk topological order, which is readily seen as arising by "gauging" the fermion parity. In other words, the microscopic symmetry that is always present for physical fermions- the conservation of their number modulo 2 -here is attributed to their $\mathbb{Z}_{2}$ gauge charge. Thus, one has an emergent $\mathbb{Z}_{2}$ gauge theory in the bulk. Additionally, there are $\mathbb{Z}_{2}$ flux loops in the bulk that are gapped. Fermions circling these flux lines pick up a $\pi$ phase shift-indicating that the fermion parity has been gauged. However, this bosonic theory is readily related to the freefermion topological phases by the following slave particle construction: Say we decompose the physical boson destruction operator into a pair of fermionic partons, $b_{r}=f_{r \uparrow} f_{r \downarrow}$. Time reversal is assumed to act projectively on the fermions: $f_{\uparrow} \rightarrow^{\mathcal{T}} f_{\downarrow} ; f_{\downarrow} \rightarrow^{\mathcal{T}}-f_{\uparrow}$. The fermions are then governed by a mean-field Hamiltonian in a topological phase: $H=$ $\sum_{i j}\left(t_{i j} f_{i}^{\dagger} f_{j}+\Delta_{i j} f_{i} f_{j}+\right.$ H.c.) (here $i$ refers to both the space and spin indices). To "ungauge" the $\mathbb{Z}_{2}$ symmetry, one simply introduces fundamental fermions $c_{r \sigma}$ in the model and condenses the pair amplitude $\left\langle\sum_{\sigma} c_{r \sigma}^{\dagger} f_{r \sigma}\right\rangle$. Now, the $\mathbb{Z}_{2}$ flux loops are confined since they have nontrivial mutual statistics with the condensate. Hence, the bulk topological order is removed, and one realizes the short-range entangled topological phase in the bulk.

\section{ABELIAN SURFACE TOPOLOGICAL ORDERS AND THE 16-FOLD WAY}

\section{A. The semion-fermion state and time-reversal symmetry}

So far, we have been discussing $\mathrm{SO}(3)_{6}$, a non-Abelian theory that we propose as a candidate for the $\mathcal{T}$-symmetric surface termination of a $\nu=1(\bmod 2)$ topological superconductor. Now, let us consider even $\nu$. In this section, we introduce an Abelian fermionic theory that we call the semion-fermion theory, which also has an anomalous realization of $\mathcal{T}$. The theory is the product of $U(1)_{2}$, which describes the universality class of bosonic fractional quantum Hall systems at $1 / 2$ filling and has quasiparticle content $\{1, s\}$, with $s$ a semion $\left(\theta_{s}=i\right)$, and the trivial fermionic theory, with quasiparticle content $\{1, f\}, f$ being a fermion. Letting $\tilde{s}=s f$, we obtain the quasiparticles $\{1, s, \tilde{s}, f\}$ with topological spins $\{1, i,-i,-1\}$ (labeled $Z_{2}^{\frac{1}{2}} \times Z_{2}^{1}$ in the notation of Ref. [29]). Potentially, one could imagine that this theory may be time-reversal symmetric if $\mathcal{T}$ exchanged $s$ and $\tilde{s}$. However, this is not possible in a $2 \mathrm{D}$ system. This time, the simple chiral central charge argument does not work. Instead, we make the following argument.

First, consider gauging the $\mathbb{Z}_{2}$ fermion parity symmetry; when this is done, the resulting theory is bosonic, and the electron carries $\mathbb{Z}_{2}$ gauge charge. It is hence described by a unitary modular tensor category, called a modular extension of the original fermionic theory. Note that a modular extension is not unique: Indeed, the trivial theory $\{1, f\}$, which has no anyons, has 16 possible modular extensions [5]. These result from the fact that even a trivial theory can have nontrivial behavior of $\pi$ fluxes-e.g., a $p+i p$ superconductor is trivial in that it has no anyons, but its modular extension - the Ising theory $\{1, \sigma, f\}$-is different from the modular extension $\{1, e, m, f\}$ of the trivial $2 \mathrm{D}$ fermionic state.

Now consider the 16 modular extensions of $\{1, s, \tilde{s}, f\}$, obtained by taking the product of $(1, s)$ with Kitaev's 16 modular extensions of $\{1, f\}$; these have chiral central charges $j / 2(\bmod 8), j=0, \ldots, 15$, and $j=0$ could potentially be $\mathcal{T}$ invariant. Since $U(1)_{2}$ has chiral central charge 1 , this $j=0$ theory is the product of $U(1)_{2}$ and the $\nu=-2$ theory of Ref. [5]; the latter has quasiparticles $\{1, a, \bar{a}, f\}$, with $a a=\bar{a} \bar{a}=f$, and $\theta_{a}=\theta_{\bar{a}}=e^{\pi i / 4}$. Let us show that there is no way that $\mathcal{T}$ could act and be consistent with the fusion rules. First, note that $\mathcal{T}$ has to fix $1, f$ and exchange $s, s f$. On the remaining quasiparticles, it must do one of two things: either $a \leftrightarrow s a$ and $\bar{a} \leftrightarrow$ $s \bar{a}$, or $\bar{a} \leftrightarrow s a$ and $a \leftrightarrow s \bar{a}$. In the first case, $s f=a(s a)$ goes to $(s a) a=s f$ under $\mathcal{T}$, which is inconsistent, and in the second case, it also goes to $(s \bar{a}) \bar{a}=s f$, which is again an inconsistency.

This argument shows that the original fermionic $\{1, s, \tilde{s}, f\}$ theory cannot exist in a $2 \mathrm{D} \mathcal{T}$-symmetric system, assuming that the above theories exhaust the modular extensions of $\{1, s, \tilde{s}, f\}$. The following argument establishes that this is so: Given any realization of $\{1, s, \tilde{s}, f\}$, and forgetting $\mathcal{T}$, we can continuously deform the Hamiltonian to any other one in this universality class. In particular, we can choose a special one, which is effectively a bosonic fractional quantum Hall layer in parallel 
with some $p+i p$ superconductor; we know that gauging this will yield one of our 16 modular extensions. Since the type of modular extension one obtains after gauging is a discrete object, it cannot change under continuous deformation. Hence, gauging any fermionic realization of $\{1, s, \tilde{s}, f\}$-including the putative $\mathcal{T}$-invariant one we started with-must yield one of the 16 above theories, none of which is $\mathcal{T}$ invariant. Hence, we have reached a contradiction.

However, it is possible to realize this theory in a $\mathcal{T}$-symmetric way on the surface of a $3 \mathrm{D}$ topological phase. Just as for the case of $\mathrm{SO}(3)_{6}$, we can define a $\mathcal{T}$ symmetry that exchanges $s \leftrightarrow \tilde{s}$ and acts with appropriate phase factors on the fusion vertices. We, once again, find that demanding $\mathcal{T}$ to commute with the $F$ and $R$ symbols requires $\mathcal{T}^{2}=-1$ on the $(s, \tilde{s}, f)$ vertices.

\section{Walker-Wang model}

Once again, we can build a Walker-Wang model based on the semion-fermion theory. Because the theory is Abelian, we can actually use a simplified cubic lattice instead of the original trivalent one here [31]. The definition of $\mathcal{T}$ symmetry also follows as in the $\mathrm{SO}(3)_{6}$ case: Haldane chains are bound to the $f$ lines, so that $f$ becomes a deconfined bulk fermion with $\mathcal{T}^{2}=-1$ : the electron. The Walker-Wang model, once again, describes the $\mathbb{Z}_{2}$-gauged version of the topological superconductor. For completeness, note that the $F$ and $R$ symbols of the semion-fermion theory are just products of those of the $\{1, f\}$ theory, in which the only nontrivial symbol is $R_{1}^{f, f}=-1$, and the $\{1, s\}$ theory, defined by $R_{1}^{s, s}=i$ and $\left[F_{s}^{s, s, s}\right]_{1,1}=-1$.

\section{Coupled-layer construction of the semion-fermion surface topological order}

We provide a coupled-layer construction of a 3D topological superconductor with a surface termination that realizes the semion-fermion Abelian topological order $\{1, f\} \times \mathrm{U}(1)_{2}=\{1, s, \tilde{s}, f\}$ with time-reversal symmetry. The building blocks are (i) a $\mathbb{Z}_{2}$ toric-code topological order with four quasiparticles, $\{1, e, m, \psi\}$, in which time reversal exchanges the two bosonic particles. This order is only possible if the fermion transforms projectively under time-reversal symmetry, as discussed in Appendix A. (ii) The second building block is a doubled-semion topological order also with four quasiparticles, $\left\{1, S, S^{\prime}, b\right\}$, where time reversal exchanges the semion and antisemion. Collecting together the action of time reversal on these excitations, we obtain

$$
e \stackrel{\mathcal{T}}{\leftrightarrow} m, \quad S \stackrel{\mathcal{T}}{\leftrightarrow} S^{\prime}, \quad \psi \stackrel{\mathcal{T}}{\leftrightarrow}-\psi .
$$

Consider a layered structure where, in each layer, a topological state of each of these two types is present (see Fig. 7). We define a unit cell as consisting of pairs

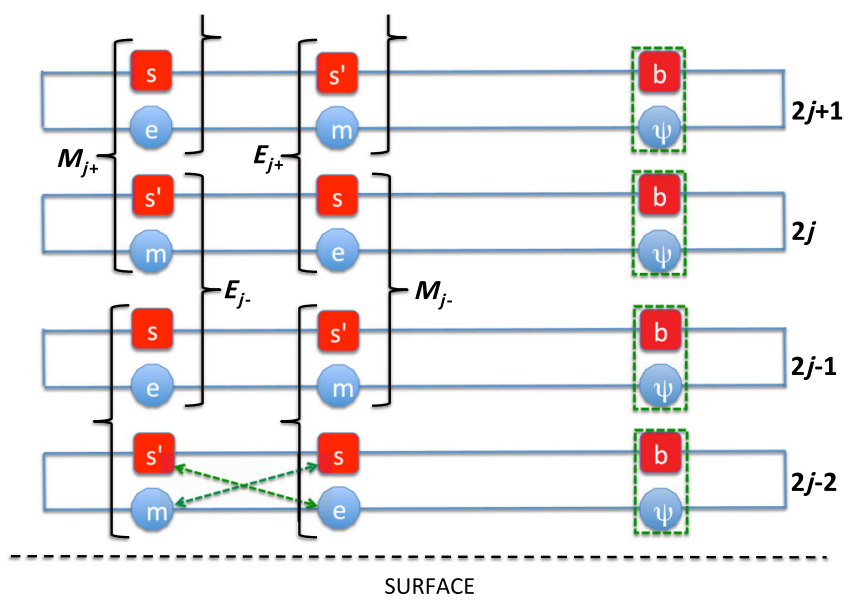

FIG. 7. Coupled-layer construction of semion-fermion topological order on the surface of a 3D topological superconductor (with even $\nu$ ). Each layer has a double-semion model and a $Z_{2}$ gauge theory. The composite excitations enclosed by the brackets can be condensed simultaneously, after which the only deconfined excitations in the bulk are electrons, $c=b \psi$, shown by the dashed green boxes. At the surface, two additional excitations emerge - shown by the dashed green lines-which are exchanged by $\mathcal{T}$. A key ingredient is that the two bosons (electric and magnetic charges) of the $Z_{2}$ gauge theory are exchanged by $\mathcal{T}$, which requires $\mathcal{T}^{2}=-1$ acting on electrons.

of these layers, and unit cell $j$ will consist of layers $2 j$ and $2 j+1$. Now, consider the four bosonic operators associated with every unit cell (we suppress the coordinates that represent the spatial position in the plane):

$$
\begin{aligned}
& E_{j+}=e_{2 j} S_{2 j} m_{2 j+1} S_{2 j+1}^{\prime}, \\
& M_{j+}=m_{2 j} S_{2 j}^{\prime} e_{2 j+1} S_{2 j+1}, \\
& E_{j-}=e_{2 j} S_{2 j} m_{2 j-1} S_{2 j-1}^{\prime}, \\
& M_{j-}=m_{2 j} S_{2 j}^{\prime} e_{2 j-1} S_{2 j-1} .
\end{aligned}
$$

It is readily seen that this set of four operators are bosonic and commute with one another. Hence, they can be simultaneously "condensed." The resulting state will confine any excitation that has nontrivial mutual statistics with these objects. In a system with periodic boundary conditions, the only excitation that has trivial mutual statistics with these four condensates is the bound state $f_{k}=\psi_{k} b_{k}$ in each layer. This is the deconfined fermion in the bulk of the system. It is readily verified that all other anyon excitations are confined in the bulk.

If, however, a surface is present, one can identify anyonic excitations that are deconfined within the surface. Consider a semi-infinite system with layers indexed by $j=0,1, \ldots, \infty$. Therefore, the condensates $E_{j=0-}$, $M_{j=0-}$ are absent. Then, the surface excitations that are now liberated (Fig. 7) are $s_{0}=e_{0} S_{0}^{\prime}$ and $\tilde{s}_{0}=m_{0} S_{0}$. Their 
combination $s_{0} \tilde{s}_{0}=e_{0} s_{0}^{\prime} m_{0} s_{0}=\psi_{0} b_{0}=f_{0}$ is just the fermion in the theory. Also, note that, using Eq. (5), under time-reversal symmetry,

$$
s_{0} \stackrel{\mathcal{T}}{\leftrightarrow} \tilde{s}_{0} .
$$

This result is exactly the surface topological order that we sought. Moreover, it transforms under time reversal in exactly the way we require.

We note a couple of important points that have been alluded to while discussing the alternate Walker-Wang construction. First, as discussed in the Appendix, the transformation law for fermions $\psi$, and hence for $f$, requires that $\mathcal{T}^{2}=-1$ when acting on these objects. Second, we note that the models used here are entirely bosonic-i.e., they do not contain microscopic fermion excitations with trivial statistics with all other particles, which can be identified with electrons. Although there are fermionic excitations $f$ that are deconfined in the bulk, these are emergent fermions and hence have mutual statistics with a $\mathbb{Z}_{2}$ flux loop excitation. This result leads to bulk $\mathbb{Z}_{2}$ topological order. To rectify this and produce a fermionic model free of bulk topological order, we introduce electron operators $c_{\sigma}$ that transform as Kramers pairs under timereversal symmetry. Then, one can condense the combination $c^{\dagger} f$, which is a boson that transforms trivially under time-reversal symmetry, since both components are Kramers pairs. As a consequence, flux excitations in the bulk of the system are confined since they have mutual statistics with this condensate, and there is no remaining bulk topological order. Now, the $f$ fermion may be interpreted as the physical electron.

\section{B. Connecting the Abelian semion-fermion state to free-fermion TScs}

In this section, we discuss a physical argument that links the Abelian semion-fermion state with the free fermion $\nu=2 \mathrm{TSc}$, with a pair of Majorana cones on the surface. Our key idea is to exploit a larger symmetry that is allowed by this problem-a U(1) symmetry that emerges upon allowing for rotations between the fermions in the pair of Majorana modes, to constrain the surface topological order. One may then constrain a candidate surface topological order by requiring it to satisfy a number of physical criteria. The resulting topological order [that respects the additional U(1) symmetry] that we conjecture is the same as the T-Pfaffian that was discussed in the context of topological-insulator (TI) surface topological order. However, here, there are important differences from the case of TIs, since time reversal is combined differently with charge conservation. While in the case of TIs the charge is left invariant under $\mathcal{T}$, here the charge will change sign under time reversal. Therefore, "charge" here should be viewed more appropriately as a component of spin (e.g., $S_{z}$ ). Technically, this means that for the case of the insulators, the symmetry is $U(1) \rtimes \mathcal{T}$, while here it is
$U(1) \times \mathcal{T}$. On destroying the artificial $U(1)$ symmetry by condensing an appropriate excitation, we are left with the Abelian semion-fermion model, which provides evidence for our assertion of the connection with the $\nu=2 \mathrm{TSc}$. A more systematic derivation of the connection to freefermion TScs will appear in a forthcoming publication.

Consider the free-fermion surface state of a $\nu=2$ topological superconductor:

$$
\mathcal{H}=\sum_{a=1}^{2} \chi_{a}^{T}\left(p_{x} \sigma_{x}+p_{y} \sigma_{z}\right) \chi_{a} .
$$

Here, $\chi_{a}^{T}=\left(\chi_{a}^{\dagger}, \chi_{a}^{\downarrow}\right)$ are two-component Majorana fields, and $\sigma$ are the Pauli matrices in the usual representation that act on the Majorana indices. The flavor index $a=1, \ldots, \nu$. In other words, , there are $\nu$ "right-handed" Majorana cones on the $2 \mathrm{D}$ surface.

The time-reversal symmetry transformation is

$$
\chi_{a}^{\dagger} \stackrel{\mathcal{T}}{\rightarrow} \chi_{a}^{\downarrow} \quad \text { and } \quad \chi_{a}^{\downarrow} \stackrel{\mathcal{T}}{\rightarrow}-\chi_{a}^{\uparrow}
$$

which leaves the Hamiltonian in Eq. (11) invariant. The $O(2)=U(1)$ symmetry is obtained by rotating between the two flavors, so the complex fermions

$$
\psi_{\sigma}=\chi_{1}^{\sigma}-i \chi_{2}^{\sigma}
$$

transform as $\psi_{\sigma} \rightarrow e^{i \varphi} \psi_{\sigma}$ under the $\mathrm{U}(1)$ rotation. In these variables, the Hamiltonian 11 is

$$
\mathcal{H}=\psi^{\dagger}\left(p_{x} \sigma_{x}+p_{y} \sigma_{z}\right) \psi
$$

where the Pauli matrices act on the spin $\sigma$ indices, which are suppressed. This is exactly the dispersion of the Dirac surface state of a topological insulator. The important difference, though, is the transformation under time-reversal symmetry:

$$
\psi_{\uparrow} \stackrel{\mathcal{T}}{\rightarrow} \psi_{\downarrow}^{\dagger} \quad \text { and } \quad \psi_{\downarrow} \stackrel{\mathcal{T}}{\rightarrow}-\psi_{\uparrow}^{\dagger} .
$$

Thus, the $\mathrm{U}(1)$ charge is reversed under $\mathcal{T}$. Moreover, the Cooper-pair operator, defined as $e^{i \Phi_{\text {Cooper }}}=\psi_{\uparrow}^{\dagger} \psi_{\downarrow}^{\dagger}$, transforms under time-reversal symmetry as $\Phi_{\text {Cooper }} \rightarrow$ $\Phi_{\text {Cooper }}+\pi$. This transformation implies that a Cooperpair condensate automatically breaks time-reversal symmetry in this case. Thus, there are no gapping terms for this surface at the quadratic level that preserve $\mathcal{T}$, as expected for the TSc surface.

Since with U(1) symmetry the $\nu=2$ TSc maps to the single Dirac-cone surface state, we can use our knowledge of the topological insulator surface topological order (STO) to guess the relevant state. The charge conserving STO, when realized purely in a $2 \mathrm{D}$ system, must break $\mathcal{T}$ symmetry and have $\sigma_{x y}=1 / 2$ and $\kappa_{x y} / T=1 / 2$, as argued for the TI surface state, by appropriately gapping the single Dirac cone with a $\mathcal{T}$-breaking mass term. Furthermore, given the presence of Majorana fermions in vortices on the surface when superconductivity is induced, 
we expect Ising topological order to be involved. The simplest such theory that has a chance of being $\mathcal{T}$ symmetric at the level of statistics is the T-Pfaffian theory $[43,44]$, a 12-particle topological order that is a subset of Ising $\times U(1)_{-8}$, where the latter is the Laughlin state $\nu_{\mathrm{QH}}=-1 / 8$ of charge $2 e$ Cooper pairs. The $U(1)_{-8}$ leads, in a $2 \mathrm{D}$ system with the same topological order, to a Hall conductance of $\sigma_{x y}=-\frac{1}{2} \frac{e^{2}}{h}$. Also, on combining with the neutral sector of the Ising theory, a simple count of all edge modes reveals $\kappa_{x y} / T=-\frac{1}{2}$, as required. The Ising $\times$ $U(1)_{-8}$ theory has 24 particles. However, an electronlike excitation with charge " $e$ " is identified, and this particle is required to have trivial statistics with all other particles, reducing the number of quasiparticles to 12 . The quasiparticles are labeled $X_{q}$, where $X \in\{I, \sigma, \psi\}$ is the Ising part and $q=0, \ldots, 7$ is the $U(1)_{-8}$ part, and they carry a charge $2 e \frac{q}{8}$. The electron is identified as $\psi_{4}$, and the locality of this excitation restricts $q$ to be even in $\left\{I_{q}, \psi_{q}\right\}$ particles and odd in the $\left\{\sigma_{q}\right\}$. Note, here, that the charge is reversed under $\mathcal{T}$; i.e., $q \rightarrow-q$ under $\mathcal{T}$.

The excitations left invariant by time-reversal symmetry are then $I_{0}, \psi_{0}, I_{4}, \psi_{4}$, which can be assigned $\mathcal{T}^{2}$ values. In addition, $I_{2}, \psi_{2}$ are mapped to $\psi_{6}, I_{6}$ under $\mathcal{T}$, differing from the first pair by an electron, which is a local excitation, allowing for the assignment of a $\mathcal{T}^{2}$ value to the pair. Indeed, $\mathcal{T}$ is fermionic when acting on the pair $\left(I_{2}, \psi_{6}\right)$, and therefore, $\mathcal{T}^{2}= \pm i(-1)^{F}$; details of this projective representation of fermion parity and time-reversal symmetries are worked out in detail in the next section, in the context of the semion-fermion model. Finally, since $\sigma_{k} \rightarrow \sigma_{-k}$, no assignment of $\mathcal{T}^{2}$ values is possible for them.

Note that $\psi_{0}$ has $\mathcal{T}^{2}=-1$ because it is a bound state of $I_{2}$ and $\psi_{6}$, which are mutual semions that are exchanged by $\mathcal{T}$ [45]. On the other hand, since $I_{4}$ is a bound state of a pair of $I_{2}$ (or $\psi_{2}$ ), it will have $\mathcal{T}^{2}=+1$. $\left[\mathcal{T}^{2}=\left(\mathcal{T}_{1} \mathcal{T}_{2}\right)\left(\mathcal{T}_{1} \mathcal{T}_{2}\right)=-\mathcal{T}_{1}^{2} \mathcal{T}_{2}^{2}=-(i)(i)=1\right.$, where $\mathcal{T}_{1 / 2}$ are the fermionic local actions of $\mathcal{T}$ near the two copies of $I_{2}$. This is elaborated in the following section.] Thus, $I_{4}$ is a charge $e$ boson with $\mathcal{T}^{2}=+1$. Since $\psi_{4}$ differs from $I_{4}$ by $\psi_{0}$, it must have $\mathcal{T}^{2}=-1$, consistent with its identification as an electron.

We can now consider breaking the $\mathrm{U}(1)$ charge conservation to obtain a simpler STO for $\nu=2 \mathrm{TSc}$. Consider condensing $I_{4}$. The consequence is that the $\sigma_{k}$ are confined. The remaining theory contains $\psi_{2 \tilde{k}}$, $I_{2 \tilde{k}}$, where $\tilde{k}=0,1$. (The particles with $\tilde{k}=2,3$ are obtained from these by combining with $I_{4}$, which is condensed.) This is just the Abelian semion-fermion theory $\{1, s\}\{1, f\}$ discussed above. The two possibilities $\nu= \pm 2$ are naturally associated with the $\mathcal{T}^{2}$ assignments of the semion (which is possible since it differs from the antisemion by a local excitation, the electronsee the next section). Here, those two assignments are related to the corresponding transformation law for $I_{2}$.
This argument links the surface of a $\nu= \pm 2$ TSc with the Abelian semion-fermion state.

\section{Remaining Abelian topological orders and the 16-fold way}

One subtlety that we have glossed over for the semionfermion theory is the value of $\mathcal{T}^{2}$ for $s$ and $\tilde{s}$. At first, one might think that there should not be a well-defined value for $\mathcal{T}^{2}$ for these anyons, since they are not fixed under $\mathcal{T}$. However, because they only differ by an electron that is a local (albeit fermionic) degree of freedom, we should really think of $s$ and $\tilde{s}$ as odd and even sectors of the same deconfined quasiparticle. Then, $\mathcal{T}^{2}=e^{i \phi}(-1)^{F}$ must hold locally for this quasiparticle, where $\phi$ is some phase. It is easy to see that since $\mathcal{T}$ anticommutes with $(-1)^{F}$, we must have $\phi= \pm \pi$, i.e., $\mathcal{T}^{2}= \pm i$ on $s$ and $\tilde{s}$. In other words, the value of $\mathcal{T}^{2}$ is equal to the value of the topological spin on $(s, \tilde{s})$ up to a sign $\eta= \pm 1$ that does not depend on whether we are acting on $s$ or $\tilde{s}$. Thus, the two possibilities $\eta= \pm 1$ correspond to two distinct theories. Indeed, flipping the theory corresponding to $\eta=1$ (i.e., taking the parity conjugate) gives the theory corresponding to $\eta=-1$ since such a flip reverses the topological spin but does not change the $\mathcal{T}^{2}$ eigenvalue. The theories with $\eta= \pm 1$ thus correspond to $\nu$ and $-\nu$, respectively, for some even integer $\nu$ (argued to be congruent to $2 \bmod 4$ in the next section).

Two copies of semion-fermion theory,-Consider now taking two copies of the $\eta=1$ semion-fermion theory. The result is [two copies of $U(1)_{2}$ times $\{1, f\}$ ] with time reversal again exchanging $s_{1} \leftrightarrow s_{1} f$ and $s_{2} \leftrightarrow s_{2} f$, where $s_{1}$ and $s_{2}$ are the two semions, both with the same topological spin. Then, $s_{1} s_{2}$ is a fermion with $\mathcal{T}^{2}=1$. To see this, let $\mathcal{T}_{1}$ and $\mathcal{T}_{2}$ be the local actions of time reversal near $s_{1}$ and $s_{2}$, respectively. They are both fermionic operators; i.e., they anticommute with fermion parity and hence with themselves. Then, $\mathcal{T}=\mathcal{T}_{1} \mathcal{T}_{2}$ is a local action of time reversal on $s_{1} s_{2}$. We then have

$$
\mathcal{T}^{2}=\left(\mathcal{T}_{1} \mathcal{T}_{2}\right)\left(\mathcal{T}_{1} \mathcal{T}_{2}\right)=-\mathcal{T}_{1}^{2} \mathcal{T}_{2}^{2}=-(i)^{2}=1 .
$$

Thus, this state has particles $\left\{1, f, s_{1}, s_{2}, s_{1} f, s_{2} f, B, F\right\}$, where the fermion $F=s_{1} s_{2}$ transforms as $\mathcal{T}^{2}=+1$ and the boson $B=s_{1} s_{2} f$ transforms as $\mathcal{T}^{2}=-1$.

Had we taken a copy of $\eta=1$ with a copy of $\eta=-1$, $s_{1} s_{2}$ would have been a fermion with $\mathcal{T}^{2}=-1$, and we would have been able to condense $s_{1} s_{2} f$ without breaking $\mathcal{T}$ and to eliminate all the topological order, as expected. However, in the present case, we cannot do this; in fact, the double semion-fermion theory cannot be realized in $2 \mathrm{D}$ with $\mathcal{T}$ symmetry. Based on the arguments above, we identify this topological order with the surface of a TSc with $\nu= \pm 4$.

Three-fermion topological order.-Although we could argue the nontrivialness of the double semion-fermion 
TABLE I. Summary of surface topological orders of TScs: The first row is the non-Abelian $\mathrm{SO}(3)_{6}$ topological order corresponding to the odd $\nu$ TSc, where $\mathcal{S}$ represents the non-Abelian excitation with semionic topological spin. The second row is the Abelian semion-fermion theory that we have argued is related to the $\nu= \pm 2 \mathrm{TSc}$. The third row contains two copies of the semion-fermion theory. Finally, the last row shows four copies of the semion-fermion theory, which corresponds (modulo the electron) to the threefermion topological order that is also the surface topological order of a purely bosonic topological phase with a $Z_{2}$ classification. Thus, eight copies of the semion-fermion topological order are trivial, i.e., realizable in a purely two-dimensional system with $\mathcal{T}$, which implies that interactions unravel the $\nu=16$ TSc connecting it to the trivial phase, consistent with Ref. [32]. The fermion $f$ transforms as $\mathcal{T}^{2}=-1$.

\begin{tabular}{lccc}
\hline \hline Label & Topological order & $\mathcal{T}$ action on anyons & $\mathcal{T}^{2}$ action on anyons \\
\hline$\nu=1(\bmod 2)$ & $\{1, \mathcal{S}, \mathcal{S} f, f\}$ & $\mathcal{S} \rightarrow \mathcal{S} f$ & \\
$\nu= \pm 2(\bmod 8)$ & $\{1, s\}\{1, f\}$ & $s \rightarrow s f$ & $\mathcal{T}^{2}= \pm i$ acting on $s ; \mathcal{T}^{2}=\mp i$ acting on $s f$ \\
$\nu= \pm 4$ & $\left\{1, s_{1}\right\}\left\{1, s_{2}\right\}\{1, f\}$ & $s_{1,2} \rightarrow s_{1,2} f$ & $\mathcal{T}^{2}= \pm i$ acting on $s_{1}, s_{2} ; \mathcal{T}^{2}=\mp i$ acting on $s_{1} f, s_{2} f ;$ \\
$\nu=8$ & $\left\{1, F_{1}, F_{2}, F_{3}\right\}\{1, f\}$ & $F_{i} \rightarrow F_{i}$ & $\mathcal{T}^{2}=+1$ acting on $s_{1} s_{2}$ \\
\hline \hline
\end{tabular}

theory directly, we proceed by taking two copies of this theory and showing that the result cannot be realized in $2 \mathrm{D}$ with $\mathcal{T}$ symmetry. This stronger result implies, in particular, that the double semion-fermion theory cannot be realized in $2 \mathrm{D}$ with $\mathcal{T}$ either. In this theory, $s_{1} s_{2} s_{1}^{\prime} s_{2}^{\prime}$ is a $\mathcal{T}^{2}=1$ boson and can be condensed. The nontrivial particles in the resulting theory are the bilinears $\left\{s s_{2}=\right.$ $\left.s_{1}^{\prime} s_{2}^{\prime}, s_{1} s_{1}^{\prime}=s_{2} s_{2}^{\prime}, s_{1} s_{2}^{\prime}=s_{2} s_{1}^{\prime}\right\}$ times $\{1, f\}$. The bilinears are all fermions and mutual semions, which shows that this is the three-fermion $\mathbb{Z}_{2}$ topological order times $\{1, f\}$. We can label the bilinears $\{e, m, \varepsilon\}$; the fusion and associativity rules are then the same as the toric code. Also, $\mathcal{T}^{2}=1$ for $e, m, \varepsilon$. It was argued in Ref. [22] that the three-fermion theory can be realized at the surface of an interacting bosonic SPT with $\mathcal{T}$ symmetry. In fact, we can argue that its fermionized version also corresponds to a nontrivial $\mathcal{T}$-invariant topological superconductor, as follows.

Supposing, for a contradiction, that the fermionized version could be realized in $2 \mathrm{D}$ in a $\mathcal{T}$-invariant way, we could use gauge fermion parity symmetry to obtain a modular $\mathcal{T}$-invariant theory. This would have to be the product of $\{1, e, m, \varepsilon\}$, with a modular extension of $\{1, f\}$; because the result is assumed to be $\mathcal{T}$ invariant, it must be nonchiral, forcing the modular extension of $\{1, f\}$ to be another copy $\left\{1, e^{\prime}, m^{\prime}, f\right\}$ of the three-fermion $\mathbb{Z}_{2}$ topological order. Since $\mathcal{T}^{2}=-1$ on $f$, we must have $\mathcal{T}^{2}=-1$ on one of the other nontrivial particles-say, $e^{\prime}$-while $\mathcal{T}^{2}=1$ on $m^{\prime}$. However, then we could condense the $\mathcal{T}^{2}=1$ boson $\mathrm{mm}^{\prime}$ while maintaining $\mathcal{T}$ invariance. The particles that remain after this condensation are $\mathcal{T}^{2}=-1$ self-bosons $\quad E \equiv e e^{\prime}=\varepsilon f, \quad M \equiv e f=\varepsilon e^{\prime}, \quad$ and the $\mathcal{T}^{2}=1$ fermion $\Sigma \equiv m=m^{\prime}$. However, this result is just the toric code $\{1, E, M, \Sigma\}$, with the $E$ and $M$ bosons being Kramers doublets. This case was argued to be impossible to realize in a time-reversal-invariant way purely in 2D in Ref. [18], which proposed that, instead, this topological order should be realized as the surface of the within-cohomology bosonic $\mathcal{T}$ SPT. Hence, the $\{1, e, m, \varepsilon\}$ three-fermion theory times $\{1, f\}$ cannot be realized in a $2 \mathrm{D}$ fermionic system with $\mathcal{T}$ symmetry.
The 16-fold way.-However, two identical copies of the "three-fermion" times $\{1, f\}$ theory can be condensed into the trivial phase without breaking $\mathcal{T}$. We have argued that the semion-fermion theory corresponds to $\nu=2 \bmod 4$, which puts the three-fermion theory at $\nu=8 \bmod 16$ and shows that $\nu=0 \bmod 16$ should be trivial and hence correspond to a trivial topological superconductor. This result is consistent with the recent result of Kitaev, showing that the free-fermion classification of 3D topological superconductors breaks down to $\mathbb{Z}_{16}$ [32]. Combining the Abelian topological orders we have constructed in this section with $\mathrm{SO}(3)_{6}$ allows us to construct a gapped $\mathcal{T}$-symmetric surface termination for any free-fermion topological superconductor. These results are summarized in Table I.

\section{CONCLUSION}

We have provided several examples of time-reversalsymmetric topological orders, realized on the surface of 3D gapped fermionic systems, that are impossible to realize in a purely $2 \mathrm{D}$ system with time-reversal symmetry. This result immediately implies that these phases are 3D fermionic SPT phases protected by $\mathcal{T}$. In particular, it is impossible to confine the surface states without breaking symmetry or closing an energy gap. If it were possible to do so, then one could eliminate the surface state on one face of a slab and be left with the "impossible" 2D topological order on the other. Since the slab can then be deformed into a 2D system, this result leads to a contradiction. It is natural to identify these phases with the freefermion topological superconductors protected by $\mathcal{T}$. However, we note that we have not proved this equivalence, e.g., by demonstrating that the free-fermion Majorana cone edge states can annihilate one of our surface topological orders. If this could be shown, it would eliminate the logical possibility that the phases we are describing are some yet-unknown topological superconductors of fermions, which are only realized in the presence of interactions. Potentially, a classification of interacting SPT phases of fermions in 3D could resolve 
this question, but that classification is also currently unavailable (Ref. [46] attempts to study this question in some other symmetry classes). These are important directions for future work.

However, there are several pieces of evidence that link the surface topological orders we mention with the freefermion topological superconductors. First, our topological orders are only possible if the electrons transform as $\mathcal{T}^{2}=-1$. This transformation is also a requirement to realize the class-DIII free-fermion topological phases. Next, it is well known that odd- $\nu$ topological superconductors have an odd number of chiral Majorana modes bound to surface domain walls between domains of opposite $\mathcal{T}$ breaking. Indeed, this is what we would find for the $\mathrm{SO}(3)_{6}$ surface if the topological order is removed while breaking $\mathcal{T}$. Imagine placing a $2 \mathrm{D}$ realization of the same topological order on the surface to obtain a quantum double model that can be confined to completely destroy topological order. Of course, in this process, one breaks $\mathcal{T}$ symmetry by the choice of the 2D topological order. If this is done in opposite ways, at the domain wall one expects an odd number of Majorana modes since the edge central charge of $\mathrm{SO}(3)_{6}$ is $c_{-}= \pm 2 \frac{1}{4}$. The difference between these two opposite values is consistent with the odd number of Majorana modes at the surface domain wall. Given the simple nature of the topological order in this case, it is tempting to attribute this to $\nu=1$, although a proof of this requires a way to bridge these different descriptions. Similarly, if the semion-fermion topological order describes a 3D free-fermion topological superconductor, it must correspond to even $\nu$ since it is compatible with a gapped edge when realized in 2D. According to the arguments above, the semion-fermion topological order corresponds to $\nu=2 \bmod 4$. Similarly, the double semion-fermion theory corresponds to $\nu=4 \bmod 8$, and the "three-fermion" times $\{1, f\}$ theory to $\nu=8 \bmod 16$. Taken together, these would then generate topological terminations for all possible 3D free-fermion $\mathcal{T}$-invariant topological superconductors.

One can also discuss analogous surface terminations for topological insulators. Shortly after this work appeared, several papers proposing such terminations were submitted $[43,44,47,48]$. In the future, we hope to connect our work on topological surface terminations with other nonperturbative definitions of the surface of fermionic SPT phases. Finally, we wish to emphasize the remarkable fact that, in some cases, the fermionic SPT phase provides a guarantee of non-Abelian topological order-if the surface of a $\nu=1$ topological superconductor is found to be gapped and $\mathcal{T}$ symmetric, it must contain non-Abelian excitations.

\section{ACKNOWLEDGMENTS}

X.C. is supported by the Miller Institute for Basic Research in Science at UC Berkeley. X.C. thanks A. Kitaev, Z. Wang, F. Burnell, P. Bonderson, T. Senthil,
C. Wang, and A. Potter for discussions. A. V. thanks A. Kitaev and P. Bonderson for insightful discussions, and is supported by ARO MURI Grant No. W911NF-12-0461. L. F. thanks Z. Wang, M. P. Fisher, and M. Metlitski for useful discussions.

\section{APPENDIX A: $\mathbb{Z}_{2}$ TOPOLOGICAL ORDER IN WHICH TIME REVERSAL EXCHANGES ELECTRIC AND MAGNETIC CHARGES}

Consider the toric-code model with the $\mathbb{Z}_{2}$ topological order, leading to four particles $\{1, e, m, \psi\}$, where the first particle is the identity, the next two are bosons (electric and magnetic charges), and the last is a fermion. The last three particles all have mutual semionic statistics with one another. Consider the action of time-reversal symmetry $\mathcal{T}$. The quasiparticles can transform projectively, and we can choose a pair of them to transform as $\mathcal{T}^{2}=-1$. Let one of these particles be the fermion $\psi$. The $\psi$ fermions are gapped in this topological phase. Consider modifying their dispersion so that they undergo a quantum phase transition into a topological superconductor (class DIII) for which there is a $\mathbb{Z}_{2}$ classification in $2 \mathrm{D}$. Now, we can investigate how the excitations transform under time-reversal symmetry. The fermions $\psi$ still transform as $\mathcal{T}^{2}=-1$. The electric and magnetic particles can both be interpreted as the $\pi$ flux for the fermions. If we choose the electric particle, we can label the object that is obtained by attaching it to the fermion as the magnetic particle; i.e., we use the fusion rule $\psi \times e=m$. In the language of the freefermion topological superconductor, if we call the $\pi$ flux the electric particle, then the magnetic particle has the opposite fermion parity. Now, it is readily seen that under time-reversal symmetry, $\stackrel{\stackrel{\mathcal{T}}{\leftrightarrow} m}{m}$. This result is related to the fact that fermion parity and time reversal anticommute [49] when acting on a $\pi$ flux in this phase, $\left[\mathcal{T}(-1)^{N_{f}}=\right.$ $\left.-(-1)^{\mathrm{N}_{\mathrm{f}}} \mathcal{T}\right]$. This anticommutation can be seen by considering the class-DIII topological superconductor as a tensor product of up-spin and down-spin electrons in a $p_{x}+i p_{y}$ and a $p_{x}-i p_{y}$ state, respectively. Since time reversal exchanges these two systems, they remain invariant. But a $\pi$ flux inserted through both superconductors will trap a pair of Majorana zero modes $\gamma_{\uparrow, \downarrow}$. Under time reversal, we have $\gamma_{\uparrow} \stackrel{\mathcal{T}}{\leftrightarrow} \gamma_{\downarrow}$ and $\gamma_{\downarrow} \stackrel{\mathcal{T}}{\stackrel{\leftrightarrow}{\leftrightarrow}}-\gamma_{\uparrow}$. This result ensures that the fermion parity $i \gamma_{\uparrow} \gamma_{\downarrow}$ for the vortex changes sign under time-reversal symmetry.

We note that although this symmetry transformation can be achieved in 2D, it cannot be described by the $K$-matrix formulation of Abelian topological orders, despite the underlying topological order being an Abelian one, specifically, that of the $\mathbb{Z}_{2}$ toric code. Indeed, in a recent classification of symmetry enhanced topological orders, using the $K$-matrix technique, this state was not produced [50]. Here, time-reversal symmetry exchanges the electric and magnetic particles, which have mutual 
statistics. In general, we can show that when time-reversal symmetry is implemented within the $K$ matrix, it cannot exchange anyons with nontrivial mutual statistics.

The proof is as follows. Consider the $K$ matrix of a timereversal-invariant state. This matrix is an even-dimensional $(2 N \times 2 N)$ symmetric matrix that can be diagonalized and brought into the form

$$
K=\sum_{\alpha=1}^{N} \lambda_{\alpha}\left[L_{\alpha} L_{\alpha}^{T}-R_{\alpha} R_{\alpha}^{T}\right] .
$$

Since this is a symmetric matrix, the eigenvalues and eigenvectors are real. The pairing of eigenvalues results from the fact that the signs of the eigenvalues $\lambda$ refer to the chirality of edge modes that should be coupled into timereversal-symmetric nonchiral pairs. Hence, for every left mover, there must be a right mover that is related by time reversal, $L_{\alpha} \stackrel{\mathcal{T}}{\leftrightarrow} R_{\alpha}$. Now, consider a quasiparticle represented by the integer vector $l$ and its time-reversed partner $\tilde{l}$. Now, expanding them in terms of the eigenstates,

$$
l=\sum_{\alpha=1}^{N}\left[a_{\alpha} L_{\alpha}+b_{\alpha} R_{\alpha}\right],
$$

where the coefficients are real numbers. Using the transformation of eigenvectors under time-reversal symmetry, we can write the time-reversed partner as

$$
\tilde{l}=\sum_{\alpha=1}^{N}\left[b_{\alpha} L_{\alpha}+a_{\alpha} R_{\alpha}\right] .
$$

Now, it is readily verified that the two quasiparticles have trivial mutual statistics since

$$
l^{T} K^{-1} \tilde{l}=0 .
$$

\section{APPENDIX B: STABILITY OF 3D BOSONIC SPT PHASES IN THE PRESENCE OF FERMIONS}

Recently, symmetry-protected topological phases of bosons were classified-for example, with just time-reversal $\mathcal{T}$ symmetry (the bosonic analog of the class-DIII systems discussed here), it was found that there were three nontrivial phases composed together in a $\mathbb{Z}_{2} \times \mathbb{Z}_{2}$ structure. The nontrivial phases are generated by (i) a group cohomology state and another state (ii) based on Kitaev's $\mathrm{E}_{8}$ state that lies outside the "group cohomology" classification.

When discussing bosonic SPT phases, one assumes that the bosons are fundamental particles. However, we could consider the possibility that the bosons are composites (like spins or Cooper pairs) of fermions. In this case, there will also be gapped fermions, in addition to the bosonic degrees of freedom. Sometimes, the bosonic topological phase can be unwound in the presence of fermions, or it can be related to one of the free-fermion topological phases. Several instances of this type were discussed in the context
TABLE II. The group-cohomology-state surface-topological order with $v_{1,2}$, which transforms as $\mathcal{T}^{2}=-1$ attached to electrons. The \pm value is the action of $\mathcal{T}^{2}$ on that quasiparticle.

\begin{tabular}{lcccc}
\hline \hline Label & 0 & 1 & 2 & 3 \\
\hline $\mathrm{B}$ & $\mathbf{1}+$ & $v_{1}-$ & $\boldsymbol{v}_{2}-$ & $f \psi-$ \\
$\mathrm{F}$ & $\psi-$ & $\boldsymbol{v}_{1} \psi+$ & $\boldsymbol{v}_{2} \psi+$ & $f+$ \\
\hline \hline
\end{tabular}

TABLE III. The beyond-group-cohomology state and the related three-fermion surface topological order, attached to electrons. The \pm value is the action of $\mathcal{T}^{2}$ on that quasiparticle.

\begin{tabular}{lcccc}
\hline \hline Label & 0 & 1 & 2 & 3 \\
\hline $\mathrm{B}$ & $\mathbf{1}+$ & $f_{1} \psi-$ & $f_{2} \psi-$ & $f_{3} \psi-$ \\
$\mathrm{F}$ & $\psi-$ & $f_{1}+$ & $f_{2}+$ & $f_{3}+$ \\
\hline \hline
\end{tabular}

of interacting fermionic SPT phases in 2D. Here, we will discuss the 3D systems, with special reference to the topological superconductors.

A useful tool to discuss this question in the $3 \mathrm{D}$ case is the topologically ordered surface termination of the 3D bosonic SPT phases. Based on this tool, we will argue that the $\mathbb{Z}_{2} \times \mathbb{Z}_{2}$ classification of bosonic SPTs is reduced to a single $\mathbb{Z}_{2}$ in the presence of fermions. Let us begin by discussing the surface-topological orders for the two bosonic SPT states, which are (i) the three-fermion state, with quasiparticles $\left\{1, f_{1}, f_{2}, f_{3}\right\}$, where the three fermions have mutual semionic statistics and transform with $T^{2}=+1$ under time reversal, and (ii) the $\mathbb{Z}_{2}$ toric-code topological order $\left\{1, v_{1}, v_{2}, f\right\}$, where the two bosonic excitations $v_{1}$, $v_{2}$ both transform as $T^{2}=-1$ Kramers doublets.

We argue that this classification collapses to a single $\mathbb{Z}_{2}$ in the presence of fermions $\psi$ that transform as $\mathcal{T}^{2}=-1$. To see this, consider putting together the fundamental fermion $\{1, \psi\}$ with these topological orders. In each case we obtain the results shown in Tables II and III.

Clearly, the Tables II and III are identical-we can represent the particles as $B_{0} \ldots B_{3}, F_{0} \ldots F_{3}$, and they have identical self-statistics and mutual statistics as well as transformation laws under time reversal. Therefore, these topological orders are identical in the presence of fundamental fermions that transform under time-reversal symmetry as $\mathcal{T}^{2}=-1$. In other words, some of the bosonic SPT phases "unwind" in the presence of fundamental fermions [51].

\section{APPENDIX C: HAMILTONIAN FOR DECORATED WALKER-WANG MODEL}

In this section, we describe in detail the Hamiltonian for our exactly solved model of a SPT realizing a gapped surface with $\mathrm{SO}(3)_{6}$ topological order. As discussed in Sec. II D, this is a decorated bosonic Walker-Wang model and is conjectured to describe the universality class of the $\nu=1$ topological superconductor, after $\mathbb{Z}_{2}$ fermion parity 
has been gauged. Although we ultimately want such a bosonic model to be built out of Kramers singlets, we first introduce auxiliary degrees of freedom, which are Kramers doublets-i.e., spin 1/2's-and write a Hamiltonian for this model. As described in Sec. IID, there will be two spin $1 / 2$ 's per link, one associated with each endpoint vertex - together, these form a four-dimensional Kramers singlet Hilbert space $\mathcal{H}_{l}^{\text {spin }}$, where $l$ labels the link. Thus, the total Hilbert space associated with each link is $\mathcal{H}_{l}^{W W} \otimes$ $\mathcal{H}_{l}^{\text {spin }}$; the Hilbert space of the model is the tensor product of these spaces over all links.

The Hamiltonian has the general form

$$
H=H_{\text {const }}+H_{\text {plaq }}
$$

The first term is

$$
H_{\text {const }}=V_{\text {fusion }}+V_{\text {links }}+V_{\text {spins }}+V_{\text {vert }}
$$

where $V_{\text {fusion }}$ is the vertex-fusion-rule-enforcing potential energy term present in the standard Walker-Wang model, $V_{\text {links }}$ is a potential-energy term preferring singlets along links not labeled with $e, V_{\text {spins }}$ is an energetic preference for spin up or down at a $(s, \tilde{s}, e)$ vertex [depending on the sign of the permutation that takes $(s, \tilde{s}, e)$ into a counterclockwise labeling of the three links adjoining the vertex], and $V_{\text {vert }}$ is a term that acts on any vertex adjoined by exactly two $e$ lines by forcing the spin 1/2's corresponding to the $e$ 's into a singlet. All of these terms will give an energy penalty of the same order $V$ to configurations that violate the constraints.

Below, we will describe the plaquette terms $H_{\text {plaq }}$ that move between different string-net and spin configurations. These terms will be modifications of the Walker-Wang plaquette terms; in particular, they will be defined to act as 0 on configurations that violate any of the constraints in $H_{\text {const }}$ in a neighborhood of the plaquette in question (i.e., on any of the links and vertices in Fig. 5). Furthermore, they will commute with each other and with all the terms in $H_{\text {const }}$. We will construct a state that satisfies all of the constraints and is the lowest eigenvalue state of all of the plaquette terms - this will be our ground state, and it will have the form described in Sec. IID, as desired. Furthermore, any other ground state must also satisfy all of the constraints, and since the spin part of a configuration that satisfies all the $H_{\text {const }}$ constraints is uniquely determined by the anyon labels, this model must have the same ground-state degeneracy as the original Walker-Wang model-i.e., it is nondegenerate, according to arguments along the lines of Ref. [22].

We still need to construct $H_{\text {plaq. }}$. We have

$$
H_{\text {plaq }}=\sum_{P} \tilde{B}_{P}^{s}
$$

where the sum is over plaquettes $P$, and $\tilde{B}_{P}^{s}$ is an "improved" Walker-Wang plaquette term. To describe this term, first recall the original Walker-Wang plaquette term $B_{P}^{s}$ defined in Eq. (4), which gives its matrix elements between fusion-rule-respecting string-net configurations (by definition, it is 0 when acting on nonfusion-rule-respecting string-net configurations). Now, $\tilde{B}_{P}^{s}$ will, by definition, be 0 unless all fusion rules and all constraints in $H_{\text {const }}$ are satisfied; when they are satisfied, we know that the spin part of the wave function is uniquely determined by the string-net labeling (we have to choose a fixed sign convention for all of the spin singlets), so we can define $\tilde{B}_{P}^{s}$ to have nonzero matrix elements precisely between those states. The operators $\tilde{B}_{P}^{s}$ defined automatically commute with all the constraintsbecause they act nontrivially only on configurations that satisfy them and move them to other configurations that satisfy them-and they commute among each other for different plaquettes $P$, for the same reason that the $B_{P}^{s}$ do. The ground state of the original Walker-Wang model, with the unique spin configuration slaved to it via the above constraints, is then also the lowest-eigenvalue state of all of the $\tilde{B}_{P}^{s}$, as desired.

[1] M.Z. Hasan and C.L. Kane, Colloquium: Topological Insulators, Rev. Mod. Phys. 82, 3045 (2010).

[2] Xiao-Liang Qi and Shou-Cheng Zhang, Topological Insulators and Superconductors, Rev. Mod. Phys. 83, 1057 (2011).

[3] M. Zahid Hasan and Joel E. Moore, Three-Dimensional Topological Insulators, Annu. Rev. Condens. Matter Phys. 2, 55 (2011).

[4] Andreas P. Schnyder, Shinsei Ryu, Akira Furusaki, and Andreas W.W. Ludwig, Classification of Topological Insulators and Superconductors in Three Spatial Dimensions, Phys. Rev. B 78, 195125 (2008).

[5] A. Kitaev, Anyons in an Exactly Solved Model and Beyond, Ann. Phys. (Amsterdam) 321, 2 (2006).

[6] X. Chen, Z.-C. Gu, and X.-G. Wen, Classification of Gapped Symmetric Phases in One-Dimensional Spin Systems, Phys. Rev. B 83, 035107 (2011).

[7] Lukasz Fidkowski and Alexei Kitaev, Topological Phases of Fermions in One Dimension, Phys. Rev. B 83, 075103 (2011).

[8] Ari M. Turner, Frank Pollmann, and Erez Berg, Topological Phases of One-Dimensional Fermions: An Entanglement Point of View, Phys. Rev. B 83, 075102 (2011).

[9] Evelyn Tang and Xiao-Gang Wen, Interacting OneDimensional Fermionic Symmetry-Protected Topological Phases, Phys. Rev. Lett. 109, 096403 (2012).

[10] X.L. Qi, A New Class of (2+1)-Dimensional Topological Superconductor with $\mathbb{Z}_{8} \quad$ Topological Classification, New J. Phys. 15, 065002 (2013).

[11] S. Ryu and S. C. Zhang, Interacting Topological Phases and Modular Invariance, Phys. Rev. B 85, 245132 (2012).

[12] Hong Yao and S. Ryu, Interaction Effect on Topological Classification of Superconductors in Two Dimensions, Phys. Rev. B 88, 064507 (2013). 
[13] Z.C. Gu and M. Levin, The Effect of Interactions on 2D Fermionic Symmetry-Protected Topological Phases with Z2 Symmetry, arXiv:1304.4569.

[14] Xie Chen, Zheng-Cheng Gu, Zheng-Xin Liu, and XiaoGang Wen, Symmetry-Protected Topological Orders in Interacting Bosonic Systems, Science 338, 1604 (2012).

[15] Xie Chen, Zheng-Cheng Gu, Zheng-Xin Liu, and Xiao-Gang Wen, Symmetry Protected Topological Orders and the Group Cohomology of Their Symmetry Group, Phys. Rev. B 87, 155114 (2013).

[16] M. Levin and Z.-C. Gu, Braiding Statistics Approach to Symmetry-Protected Topological Phases, Phys. Rev. B 86, 115109 (2012).

[17] Yuan-Ming Lu and Ashvin Vishwanath, Theory and Classification of Interacting Integer Topological Phases in Two Dimensions: A Chern-Simons Approach, Phys. Rev. B 86, 125119 (2012).

[18] Ashvin Vishwanath and T. Senthil, Physics of ThreeDimensional Bosonic Topological Insulators: SurfaceDeconfined Criticality and Quantized Magnetoelectric Effect, Phys. Rev. X 3, 011016 (2013).

[19] Cenke $\mathrm{Xu}$ Three-Dimensional Symmetry Protected Topological Phase Close to Antiferromagnetic Néel Order, Phys. Rev. B 87, 144421 (2013).

[20] M. Metlitski, C. L. Kane, and M.P. A. Fisher, Bosonic Topological Insulator in Three Dimensions and the Statistical Witten Effect, Phys. Rev. B 88, 035131 (2013).

[21] C. Wang and T. Senthil, Boson Topological Insulators: A Window into Highly Entangled Quantum Phases, Phys. Rev. B 87, 235122 (2013).

[22] F. J. Burnell, X. Chen, L. Fidkowski, and A. Vishwanath, Exactly Soluble Model of a 3D Symmetry Protected Topological Phase of Bosons with Surface Topological Order, arXiv:1302.7072.

[23] A. Kitaev, in Periodic Table for Topological Insulators and Superconductors, edited by V. Lebedev and M. Feigel'Man, American Institute of Physics Conference Series Vol. 1134 (AIP, New York, 2009), pp. 22-30.

[24] R. Balian and N. R. Werthamer, Superconductivity with Pairs in a Relative p Wave, Phys. Rev. 131, 1553 (1963).

[25] G.E. Volovik, The Universe in a Helium Droplet, International Series of Monographs on Physics (Oxford University Press, New York, 2009), ISBN 9780199564842.

[26] Gregory Moore and Nicholas Read, Nonabelions in the Fractional Quantum Hall Effect, Nucl. Phys. B 360, 362 (1991).

[27] However, a related theory, where one reverses the direction of the Majorana mode, can be made $\mathcal{T}$ invariant. Since this may have an application to 3D topological insulators, it will be discussed in a separate publication.

[28] Z. Wang (private communication).

[29] P. Bonderson, Ph.D. thesis, California Institute of Technology, 2007, http://thesis.library .caltech.edu/2447.

[30] K. Walker and Z. Wang $(3+1)-T Q F T$ s and Topological Insulators Front. Phys. 7, 150 (2012).

[31] C. W. von Keyserlingk, F. J. Burnell, and S. H. Simon Three-Dimensional Topological Lattice Models with Surface Anyons, Phys. Rev. B 87, 045107 (2013).
[32] A. Kitaev, http://online.kitp.ucsb.edu/online/topomat11/ kitaev.

[33] Edward Witten, Quantum Field Theory and the Jones Polynomial, Commun. Math. Phys. 121, 351 (1989).

[34] N. Read and E. Rezayi, Beyond Paired Quantum Hall States: Parafermions and Incompressible States in the First Excited Landau Level, Phys. Rev. B 59, 8084 (1999).

[35] Sometimes, only theories with $k=0 \bmod 4$ are labeled $\mathrm{SO}(3)_{k}$, since otherwise they are nonmodular. Since we are specifically interested in theories that contain the electron, which are necessarily nonmodular, we will not make this distinction.

[36] This structure is reminiscent of the notion of $G$ action in a braided $G$-crossed category $[37,38]$.

[37] P. Etingof, D. Nikshych, and V. Ostrik, Fusion Categories and Homotopy Theory, Quantum Topol. 1, 209 (2010).

[38] Vladimir Drinfeld, Shlomo Gelaki, Dmitri Nikshych, and Victor Ostrik, On Braided Fusion Categories I, Selecta Mathematica Sovietica 16, 1 (2010).

[39] X. Chen, Y.-M. Lu, and A. Vishwanath, Symmetry Protected Topological Phases from Decorated Domain Walls, arXiv:1303.4301.

[40] It is actually known that the symmetric center $\mathcal{Z}(\mathcal{C})$ of any braided fusion category $\mathcal{C}$ comes in two types [41]: Either it consists entirely of bosons and is isomorphic to the set of representations of some finite group $G$, in which case the bulk forms a (possibly twisted) $G$-gauge theory, or it is a supersymmetric version of this, where $G$ contains some odd elements and the corresponding representations have even and odd sectors, corresponding to bosons and fermions, respectively.

[41] S. Doplicher and J. Roberts, A New Duality for Compact Groups, Inventiones Mathematicae 98, 157 (1989).

[42] Indeed, if we take a gauge transformation to act by phase factor $\beta_{c}^{a, b}$ on the fusion space $V_{c}^{a, b}$, then any choice that satisfies $\quad \beta_{s}^{s, s} \beta_{\tilde{s}}^{\tilde{s}, \tilde{s}}=i, \quad \beta_{\tilde{s}}^{s, s} \beta_{s}^{\tilde{s}, \tilde{s}}=-i, \quad \beta_{s}^{s, \tilde{s}} \beta_{\tilde{s}}^{\tilde{s}, s}=-i$, $\beta_{s}^{\tilde{s}, s} \beta_{\tilde{s}}^{s, \tilde{s}}=-i$ and sets the other $\beta_{c}^{a, b}$ to be trivial does the trick. The action of $\mathcal{T}$ is then truly onsite.

[43] P. Bonderson, C. Nayak, and X.-L. Qi, A Time-Reversal Invariant Topological Phase at the Surface of a $3 D$ Topological Insulator arXiv:1306.3230.

[44] X. Chen, L. Fidkowski, and A. Vishwanath, Symmetry Enforced Non-Abelian Topological Order at the Surface of a Topological Insulator, arXiv:1306.3250.

[45] A pair of particles that are interchanged by $\mathcal{T}$ and have mutual statistics $\eta= \pm 1$ will, when fused together, carry $\mathcal{T}^{2}=\eta$. This may be understood by regarding the action of $\mathcal{T}^{2}$ as taking one particle around the other.

[46] Z. C. Gu and X.-G. Wen, Symmetry-Protected Topological Orders for Interacting Fermions-Fermionic Topological Non-linear Sigma-Models and a Group Super-cohomology Theory, arXiv:1201.2648.

[47] M. A. Metlitski, C.L. Kane, and M.P.A. Fisher, A Symmetry-Respecting Topologically-Ordered Surface Phase of $3 D$ Electron Topological Insulators, arXiv:1306.3286.

[48] C. Wang, A. C. Potter, and T. Senthil, Gapped Symmetry Preserving Surface-State for the Electron Topological Insulator, arXiv:1306.3238. 
[49] Xiao-Liang Qi, Taylor L. Hughes, S. Raghu, and ShouCheng Zhang, Time-Reversal-Invariant Topological Superconductors and Superfluids in Two and Three Dimensions, Phys. Rev. Lett., 102, 187001 (2009).

[50] Y.-M. $\mathrm{Lu}$ and A. Vishwanath, Classification and Properties of Symmetry Enriched Topological Phases: A
Chern-Simons Approach with Applications to Z2 Spin Liquids, arXiv:1302.2634.

[51] Related results have been obtained by C. Wang, A. Potter, and T. Senthil (unpublished) in the context of topological insulators. 